\title{
A Posteriori Error Estimate and Adaptive Mesh Refinement Algorithm for Atomistic/Continuum Coupling with Finite Range Interactions in Two Dimensions
}

\author{
Mingjie Liao ${ }^{1}$, Ping Lin $^{2}$ and Lei Zhang ${ }^{3, *}$ \\ ${ }^{1}$ School of Mathematics and Physics, University of Science and Technology Beijing, \\ Beijing 100083, P.R. China. \\ 2 Department of Mathematics, University of Dundee, Dundee, DD1 4HN, Scotland, \\ United Kingdom. \\ 3 School of Mathematical Sciences, Institute of Natural Sciences and MOE-LSC, \\ Shanghai Jiao Tong University, Shanghai 200240, P.R. China.
}

Received 27 June 2018; Accepted (in revised version) 12 December 2018

\begin{abstract}
In this paper, we develop the residual based a posteriori error estimates and the corresponding adaptive mesh refinement algorithm for atomistic/continuum (a/c) coupling with finite range interactions in two dimensions. We have systematically derived a new explicitly computable stress tensor formula for finite range interactions. In particular, we use the geometric reconstruction based consistent atomistic/continuum (GRAC) coupling scheme, which is quasi-optimal if the continuum model is discretized by $P^{1}$ finite elements. The numerical results of the adaptive mesh refinement algorithm is consistent with the quasi-optimal a priori error estimates.
\end{abstract}

AMS subject classifications: 65N12, 65N15, 70C20, 82D25

Key words: Atomistic models, coarse graining, atomistic-to-continuum coupling, quasicontinuum method, a posteriori error estimate.

\section{Introduction}

Atomistic/continuum (a/c) coupling methods are a class of computational multiscale methods for crystalline solids with defects that aim to optimally balance the accuracy of the atomistic model and the efficiency of the continuum model $[12,19,36]$. The construction and analysis of different a/c coupling methods have attracted considerable attention in the research community in recent years. Rigorous a prior analysis and systematic

*Corresponding author. Email addresses: mliao@xs.ustb.edu.cn (M. Liao), plin@maths.dundee.ac.uk (P. Lin), lzhang2012@sjtu.edu.cn (L. Zhang) 
benchmark has been done in, for example, $[13-15,23,29,30]$. We refer readers to $[16,18]$ for a review. The study of a/c coupling methods has not only provided an analytical framework for the prototypical problems [9], but also opened avenue for coupling schemes in more complicated physical situations $[3,4,10]$.

Like many multiscale methods dealing with defects or singularities, adaptivity is the key for the efficient implementation of a/c coupling methods. In contrast to the a priori analysis, the development of a posteriori analysis for a/c coupling methods are still lagging behind. Although heuristic methods have been proposed in the engineering literature $[33,36,40]$. Previous mathematical justifications are largely limited to one dimension cases $[1,2,33]$. In particular, the residual based a posteriori error bounds for a/c coupling schemes are first derived in $[20,25,27]$ by Ortner et al. in one dimension.

In [41], we carried out a rigorous a posteriori analysis of the residual, the stability constant, and the error bound, for a consistent atomistic/continuum coupling method [28] with nearest neighbor interactions in two dimensions. Corresponding adaptive mesh refinement algorithm was designed and implemented based on the a posteriori error estimates, and the convergence rate with respect to degrees of freedom is the same as quasi-optimal a priori error estimates. This is the first rigorous a posteriori analysis for a/c coupling method in two dimensions. With the a posteriori error estimates and the adaptive algorithm, we can not only automatically move the a/c interface and adjust the discretization of the continuum region, but also change the size of the computational domain. We have also introduced the so-called "stress tensor correction" technique, which distinguish the essential difference of high dimensional results compared with previous one dimensional results.

In this paper, we treat the more general case of a/c coupling with finite range interactions, which is physically more relevant and algorithmically more involved. The a priori analysis of GRAC scheme has been extended from nearest neighbor case in [28] to finite range interactions in [29]. $\ell^{1}$-minimization is introduced to resolve the issue of non-uniqueness of reconstruction parameters, and a stabilisation mechanism is proposed in [24] to reduce the stability gap between the a/c coupling scheme and the original atomistic model.

The analytical framework for both a priori analysis and a posteriori analysis of a/c coupling methods strongly relies on the stress based formulation. In [21,26], an explicit formulation of stress tensor is proposed based on a mollified version of line measure supported on the interaction bonds, thence one can obtain an integral representation of finite differences to further derive an integral representation of the first variation of the interaction energy. This representation greatly simplifies the expression of the stress tensor and plays a significant role in the a priori analysis. However, the obtained stress tensor is a function of the continuous space variable, therefore it is difficult to compute in practice, and not suitable for the a posteriori estimates and adaptive computation.

In this paper, we derive a novel expression of the stress tensor for finite range interactions, which is new to the best of our knowledge. The stress tensor is piecewise constant and only depends on a local neighborhood, therefore it is computable and the assembly 
cost is linear with respect to the number of bonds. This stress formulation allows for the convenient derivation of a posterior error estimates and efficient implementation of the adaptive algorithms.

The paper is organized as follows. We set up the atomistic to continuum (a/c) coupling models for point defects in Section 2, and introduce the general GRAC formulation in Section 3. In Section 4, we present the stress formulation and the stress tensor assembly algorithm for finite range interactions. In Section 5 the rigorous a posteriori error estimates based adaptive algorithm is deduced and complemented by numerical experiments. We draw conclusions and make suggestions for future research in Section 6. Some auxiliary results are given in the Appendix Section A.

\section{Model formulation}

In this section, We setup an atomistic model for crystal defects in an infinite lattice in the spirit of [9] in Section 2.1 and then introduce the Cauchy-Born continuum model in Section 2.2. We give a generic form of a/c coupling schemes in Section 2.3.

\subsection{Atomistic model}

\subsubsection{Atomistic lattice and defects}

Given $d \in\{2,3\}, \mathrm{A} \in \mathbb{R}^{d \times d}$ non-singular, $\Lambda^{\text {hom }}:=\mathrm{A} \mathbb{Z}^{d}$ is the homogeneous reference lattice which represents a perfect single lattice crystal formed by identical atoms. $\Lambda \subset \mathbb{R}^{d}$ is the reference lattice with some local defects. The mismatch between $\Lambda$ and $\Lambda^{\text {hom }}$ represents possible defects $\Lambda^{\text {def }}$, which are contained in some localized defect cores $D^{\text {def }}$ such that the atoms in $\Lambda \backslash D^{\text {def }}$ do not interact with defects $\Lambda^{\text {def }}$. Vacancy, interstitial and impurity are different types of possible point defects.

\subsubsection{Lattice function and lattice function space}

Given $m \in\{1,2,3\}$, denote the set of vector-valued lattice functions by

$$
\mathscr{U}:=\left\{v: \Lambda \rightarrow \mathbb{R}^{m}\right\} .
$$

A deformed configuration is a lattice function $y \in \mathscr{U}$. Let $x$ be the identity map, the displacement $u \in \mathscr{U}$ is defined by $u(\ell)=y(\ell)-x(\ell)=y(\ell)-\ell$ for any $\ell \in \Lambda$.

For each $\ell \in \Lambda$, we prescribe an interaction neighborhood $\mathcal{N}_{\ell}:=\left\{\ell^{\prime} \in \Lambda|0<| \ell^{\prime}-\ell \mid \leq r_{\text {cut }}\right\}$ with some cut-off radius $r_{\text {cut }}$. The interaction range $\mathcal{R}_{\ell}:=\left\{\ell^{\prime}-\ell \mid \ell^{\prime} \in \mathcal{N}_{\ell}\right\}$ is defined as the union of lattice vectors defined by the finite differences between lattice points in $\mathcal{N}_{\ell}$ and $\ell$. Define the "finite difference stencil" $D v(\ell):=\left\{D_{\rho} v(\ell)\right\}_{\rho \in \mathcal{R}_{\ell}}:=\{v(\ell+\rho)-v(\ell)\}_{\rho \in \mathcal{R}_{\ell}}$.

The homogeneous lattice $\Lambda^{\text {hom }}=A \mathbb{Z}^{d}$ naturally induces a simplicial micro-triangulation $\mathcal{T}^{\text {a }}$. In two dimensions, $\mathcal{T}^{\mathrm{a}}=\left\{\mathrm{A} \xi+\hat{T}, \mathrm{~A} \xi-\hat{T} \mid \xi \in \mathbb{Z}^{2}\right\}$, where $\hat{T}=\operatorname{conv}\left\{0, e_{1}, e_{2}\right\}$. Let $\bar{\zeta} \in W^{1, \infty}\left(\Lambda^{\text {hom }} ; \mathbb{R}\right)$ be the $P_{1}$ nodal basis function associated with the origin; namely, $\bar{\zeta}$ 
is piecewise linear with respect to $\mathcal{T}^{\mathrm{a}}$, and $\bar{\zeta}(0)=1$ and $\bar{\zeta}(\xi)=0$ for $\xi \neq 0$ and $\xi \in \Lambda^{\text {hom }}$. The nodal interpolant of $v \in \mathscr{U}$ can be written as

$$
\bar{v}(x):=\sum_{\xi \in \mathbb{Z}^{d}} v(\xi) \bar{\zeta}(x-\xi) .
$$

We can introduce the discrete homogeneous Sobolev spaces

$$
\mathscr{U}^{1,2}:=\left\{u \in \mathscr{U} \mid \nabla \bar{u} \in L^{2}\right\},
$$

with semi-norm $\|\nabla \bar{u}\|_{L^{2}}$.

\subsubsection{Interaction potential}

We consider the general multibody interaction potential of the generic pair functional form [39], which includes the widely used potentials such as EAM (Embedded Atom Method) potential [5] and Finnis-Sinclair model [11]. Namely, the potential is a function of the distances between atoms within interaction range and has no angular dependence. For example, for each $\ell \in \Lambda$, let $V_{\ell}(y)$ denote the site energy associated with the lattice site $\ell \in \Lambda$, the $E A M$ potential reads

$$
\begin{aligned}
V_{\ell}(y) & :=\sum_{\ell^{\prime} \in \mathcal{N}_{\ell}} \Phi\left(\left|y(\ell)-y\left(\ell^{\prime}\right)\right|\right)+F\left(\sum_{\ell^{\prime} \in \mathcal{N}_{\ell}} \psi\left(\left|y(\ell)-y\left(\ell^{\prime}\right)\right|\right)\right) \\
& =\sum_{\rho \in \mathcal{R}_{\ell}} \Phi\left(\left|D_{\rho} y(\ell)\right|\right)+F\left(\sum_{\rho \in \mathcal{R}_{\ell}} \psi\left(\left|D_{\rho} y(\ell)\right|\right)\right),
\end{aligned}
$$

with the pair potential $\Phi$, the electron density function $\psi$ and the embedding function $F$.

We assume that the potential $V_{\ell}(y) \in C^{k}\left(\left(\mathbb{R}^{d}\right)^{\mathcal{R}_{\ell}}\right), k \geq 2$. We also assume that $V_{\ell}(y)$ is homogeneous outside the defect region $D^{\text {def }}$, namely, $V_{\ell}=V$ and $\mathcal{R}_{\ell}=\mathcal{R}$ for $\ell \in \Lambda \backslash D^{\text {def }}$. Furthermore, $V$ and $\mathcal{R}$ have the following point symmetry: $\mathcal{R}=-\mathcal{R}$, and $V\left(\left\{-g_{-\rho}\right\}_{\rho \in \mathcal{R}}\right)=$ $V(g)$.

For an infinite lattice, assume the macroscopic applied strain is $\mathrm{B} \in \mathbb{R}^{d \times d}$, we redefine the potential $V_{\ell}(y)$ as the difference $V_{\ell}(y)-V_{\ell}\left(y_{\mathrm{B}}\right)$. We denote the energy functional $\mathscr{E}(y)$ as the infinite sum of the redefined potential over $\Lambda$, which is well-defined for $y-y^{\mathrm{B}} \in$ $\mathscr{U}^{1,2}[9]$,

$$
\mathscr{E}(y)=\sum_{\ell \in \Lambda} V_{\ell}(y)
$$

Under the above conditions, the goal of the atomistic problem is to find a strongly stable equilibrium $y$, such that

$$
y \in \arg \min \left\{\mathscr{E}(y) \mid y-y^{B} \in \mathscr{U}^{1,2}\right\} .
$$

$y$ is strongly stable if there exists $c_{0}>0$ such that

$$
\left\langle\delta^{2} \mathscr{E}(y) v, v\right\rangle \geq c_{0}\|\nabla v\|_{L^{2}}^{2}, \quad \forall v \in \mathscr{U}^{1,2} .
$$




\subsection{Continuum model}

From the atomistic model, a continuum model can be derived by coarse graining, and computationally it allows for the reduction of degrees of freedom when the deformation is smooth. A typical choice in the multi-scale context is the Cauchy-Born continuum model $[8,26]$. Let $W: \mathbb{R}^{d \times d} \rightarrow \mathbb{R}$ be a strain energy density function, the Cauchy-Born energy density $W$ is defined by

$$
W(\mathrm{~F}):=\operatorname{det} \mathrm{A}^{-1} V(\mathrm{~F} \cdot \mathcal{R}) .
$$

\subsection{Generic formulation of energy based atomistic/continuum coupling}

We give a generic formulation of the a/c coupling method and employ concepts and notation from various earlier works, such as $[17,19,29,36,37]$, and we adapt the formulation to the settings in this paper.

The computational domain $\Omega_{R}=\Omega_{R}^{\mathrm{a}} \cup \Omega_{R}^{\mathrm{c}} \subset \mathbb{R}^{d}$ is a simply connected, polygonal and closed set, consists of the atomistic region $\Omega_{R}^{\mathrm{a}}$ and the continuum partition $\Omega_{R}^{\mathrm{c}}$, where $R$ is the radius of $\Omega_{R}$. Given the reference lattice $\Lambda$ with some local defects, we decompose the set $\Lambda^{\mathrm{a}, \mathrm{i}}:=\Lambda \cap \Omega_{R}^{\mathrm{a}}=\Lambda^{\mathrm{a}} \cup \Lambda^{\mathrm{i}}$ into a core atomistic set $\Lambda^{\mathrm{a}}$ and an interfacial atomistic set $\Lambda^{\mathrm{i}}$ such that $\Lambda \cap D^{\mathrm{def}} \subset \Lambda^{\mathrm{a}}$, where $D^{\text {def }}$ represents the defect core. Let $\mathcal{T}_{h, R}^{\mathrm{a}}$ (respectively $\mathcal{T}_{h, R}^{\mathrm{i}}$ ) be the canonical triangulation induced by $\Lambda^{\mathrm{a}}$ (respectively $\Lambda^{\mathrm{i}}$ ), and $\mathcal{T}_{h, R}^{c}$ be a shaperegular simplicial partition of the continuum region. We denote $\mathcal{T}_{h, R}=\mathcal{T}_{h, R}^{\mathrm{c}} \cup \mathcal{T}_{h, R}^{\mathrm{i}} \cup \mathcal{T}_{h, R}^{\mathrm{a}}$ as the triangulation of the a/c coupling configuration. Please see Fig. 1 for an illustration of the computational mesh.

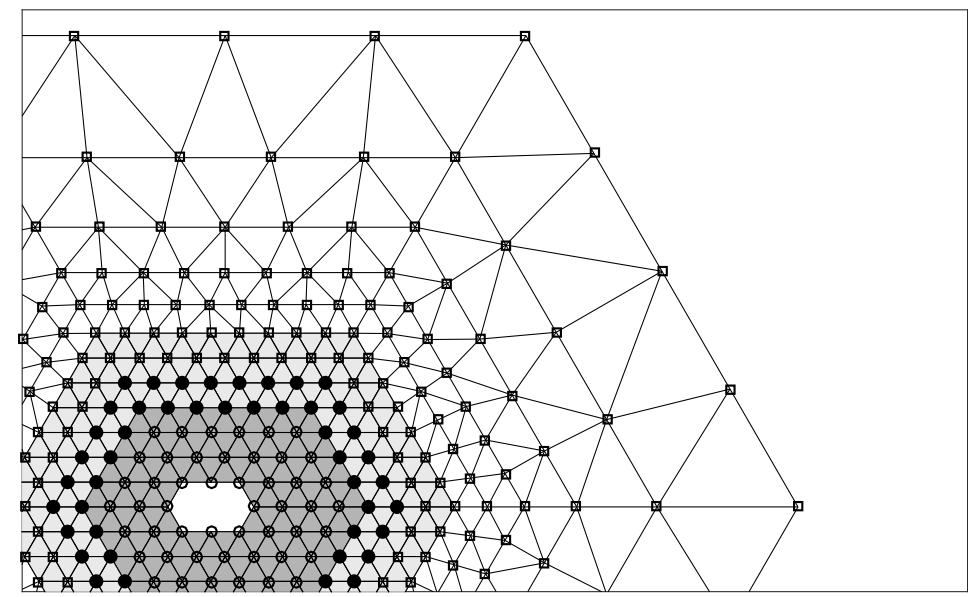

Figure 1: Illustration of computational mesh. The computational domain is $\Omega_{R}$, and the corresponding triangulation is $\mathcal{T}_{h, R}$. $\circ$ in the DimGrey region are atoms of $\Lambda^{a}$. For the next nearest neighbor interaction, $\Lambda^{\mathrm{i}}$ contains - in the LightGrey interface region. $\square$ are continuum degrees of freedom. 
The space of coarse-grained displacements is

$$
\begin{aligned}
\mathscr{U}_{h, R}:=\left\{u_{h}: \Omega_{h, R} \rightarrow \mathbb{R}^{m} \mid\right. & u_{h} \text { is continuous and p.w. affine w.r.t. } \mathcal{T}_{h, R}, \\
& \left.u_{h}=0 \text { on } \partial \Omega_{R}\right\} .
\end{aligned}
$$

The subscript $R$ in the above definitions can be dropped if there is no confusion, for example, we can replace $\mathcal{T}_{h, R}$ by $\mathcal{T}_{\mathrm{h}}$.

Let $\operatorname{vor}(\ell)$ represents the voronoi cell associated with $\ell$ of the homogeneous reference lattice $\Lambda^{\text {hom }}:=\mathrm{A} \mathbb{Z}^{d}$, for some given non-singular $\mathrm{A} \in \mathbb{R}^{d \times d}$. We have $|\operatorname{vor}(\ell)|=\operatorname{det} \mathrm{A}$, for each $\ell \in \Lambda^{\text {hom }}$. For each $\ell \in \Lambda$ denote its effective cell as $v_{\ell}$ (see [28]), let $\omega_{\ell}:=\frac{\left|v_{\ell}\right|}{|\operatorname{vor}(\ell)|}$ be the effective volume associated with $\ell$. For each element $T \in \mathcal{T}_{\mathrm{h}}$ we define the effective volume of $T$ by

$$
\omega_{T}:=\left|T \backslash\left(\bigcup_{\ell \in \Lambda^{\mathrm{a}}} \operatorname{vor}(\ell)\right) \backslash\left(\bigcup_{\ell \in \Lambda^{\mathrm{i}}} v_{\ell}^{\mathrm{i}}\right)\right| \cdot
$$

We note that $\omega_{T}=0$ if $T \in \mathcal{T}_{h}^{\mathrm{a}} \backslash \mathcal{T}_{h}^{\mathrm{i}}, \omega_{T}=|T|$ if $T \in \mathcal{T}_{h}^{\mathrm{c}} \backslash \mathcal{T}_{h}^{\mathrm{i}}$, and $0 \leq \omega_{T}<|T|$ if $T \in \mathcal{T}_{h}^{\mathrm{i}}$, depends on how we defining $v_{\ell}^{i}$, the effective cell of $\ell \in \Lambda^{i}$. The choices of $v_{\ell}$ and $\omega_{T}$ satisfy $\sum_{\ell \in \Lambda^{\mathrm{a}, \mathrm{i}}} v_{\ell}+\sum_{T \in \mathcal{T}_{\mathrm{h}}} \omega_{T}=\left|\Omega_{h, R}\right|$.

Now we are ready to define the generic a/c coupling energy functional $\mathscr{E}$,

$$
\mathscr{E} \mathrm{h}\left(y_{h}\right):=\sum_{\ell \in \Lambda^{\mathrm{a}}} V_{\ell}\left(y_{h}\right)+\sum_{\ell \in \Lambda^{\mathrm{i}}} \omega_{\ell} V_{\ell}^{\mathrm{i}}\left(y_{h}\right)+\sum_{T \in \mathcal{T}_{\mathrm{h}}} \omega_{T} W\left(\left.\nabla y_{h}\right|_{T}\right),
$$

where $V_{\ell}^{\mathrm{i}}$ is a modified interface site potential.

The goal of a/c coupling is to find

$$
y_{h, R} \in \operatorname{argmin}\left\{\mathscr{E}^{\mathscr{h}}\left(y_{h}\right) \mid y_{h}-y^{B} \in \mathscr{U}_{h, R}\right\} .
$$

The subscript $R$ in $y_{h, R}$ and $\mathscr{U}_{h, R}$ can be omitted if there is no confusion.

The first variation of the a/c coupling variational problem (2.5) is to find $y_{h}-y^{\mathrm{B}} \in \mathscr{U}_{h, R}$ such that

$$
\left\langle\delta \mathscr{E}^{\mathscr{h}}\left(y_{h}\right), v_{h}\right\rangle=0, \quad \forall v_{h} \in \mathscr{U}_{h, R} .
$$

Spurious artificial force could occur at the interface for energy based coupling even for homogeneous deformation [36], and was dubbed "ghost force". The issue of "ghost force removal" has received considerable attention in the recent years, and consistent a/c coupling methods without ghost force were developed by $[7,37]$ in one dimension and $[28,34]$ in two dimensions. We will introduce the consistent GRAC formulation in Section 3.

\section{General GRAC formulation}

In this section, we describe the construction of the geometric reconstruction based consistent atomistic/continuum (GRAC) coupling enersec:gracgy for multibody potentials with general interaction range and arbitrary interfaces. 
Given the homogeneous site potential $V(D y(\ell))$, we can represent the interface potential $V_{\ell}^{\mathrm{i}}$ in (2.4) in terms of $V$. For each $\ell \in \Lambda^{\mathrm{i}}, \rho, \zeta \in \mathcal{R}_{\ell}$, let $C_{\ell ; \rho, \zeta}$ be free parameters, and define

$$
V_{\ell}^{\mathrm{i}}(y):=V\left(\left(\sum_{\varsigma \in \mathcal{R}_{\ell}} C_{\ell ; \rho, \zeta} D_{\varsigma} y(\ell)\right)_{\rho \in \mathcal{R}_{\ell}}\right)
$$

A convenient short-hand notation is

$$
V_{\ell}^{\mathrm{i}}(y)=V\left(C_{\ell} \cdot D y(\ell)\right), \quad \text { where }\left\{\begin{array}{l}
C_{\ell}:=\left(C_{\ell ; \rho, \zeta}\right)_{\rho, \zeta \in \mathcal{R}_{\ell}} \\
C_{\ell} \cdot D y:=\left(\sum_{\varsigma \in \mathcal{R}_{\ell}} C_{\ell ; \rho, \zeta} D_{\zeta} y\right)_{\rho \in \mathcal{R}_{\ell}} .
\end{array}\right.
$$

We call the parameters $C_{\ell ; \rho, \zeta}$ as the reconstruction parameters.

To construct consistent a/c coupling energy, we need to enforce the so-called patch tests for the energy functional $\mathscr{E}$, namely, energy patch test (3.2) and force patch test (3.4). Those patch tests in turn prescribe conditions (3.3) and (3.6) for the reconstruction parameters $C$. In general, the reconstruction parameters satisfying patch tests are not unique, an $\ell^{1}$-minimization technique can be introduced to choose the "optimal" parameters [29]. Also, a stabilisation mechanism can be applied to improve the stability of the GRAC coupling scheme [24].

\subsection{Energy patch test}

To guarantee that $\mathscr{E}^{\mathscr{h}}$ approximates the atomistic energy $\mathscr{E}=\sum_{\ell \in \Lambda} V_{\ell}(y)$, it is reasonable to require that the interface potentials satisfy an energy patch test

$$
V_{\ell}^{\mathrm{i}}\left(y^{\mathrm{F}}\right)=V\left(y^{\mathrm{F}}\right), \quad \forall \ell \in \Lambda^{\mathrm{i}}, \quad \mathrm{F} \in \mathbb{R}^{m \times d},
$$

namely, the interface potential coincides with the atomistic potential for uniform deformations.

For the GRAC coupling scheme, a sufficient and necessary condition for the energy patch test is that $\mathrm{F} \cdot \mathcal{R}(\ell)=C_{\ell} \cdot(\mathrm{F} \cdot \mathcal{R})$ for all $\mathrm{F} \in \mathbb{R}^{m \times d}$ and $\ell \in \Lambda^{\mathrm{i}}$. This is equivalent to

$$
\rho=\sum_{\varsigma \in \mathcal{R}(\ell)} C_{\ell ; \rho, \zeta \zeta,} \quad \forall \ell \in \Lambda^{\mathrm{i}}, \quad \rho \in \mathcal{R}(\ell) .
$$

\subsection{Force patch test}

We call the following condition the force patch test, namely, for $\Lambda=\Lambda^{\text {hom }}$ and $\Phi_{\ell}=\Phi$,

$$
\left\langle\delta \mathscr{E}^{\mathrm{h}}\left(y^{\mathrm{F}}\right), v_{h}\right\rangle=0 \quad \forall v_{h} \in \mathscr{U}_{h}, \quad \mathrm{~F} \in \mathbb{R}^{m \times d},
$$

where $\mathrm{F}$ is some uniform deformation gradient. This is saying that there is no artificial "ghost force" for uniform deformations.

From the general GRAC formulation (2.4), we can decompose the first variation of the a/c coupling energy into three parts,

$$
\left\langle\delta \mathscr{E}^{\mathrm{h}}\left(y^{\mathrm{F}}\right), v_{h}\right\rangle=\left\langle\delta \mathscr{E}^{\mathrm{a}}\left(y^{\mathrm{F}}\right), v_{h}\right\rangle+\left\langle\delta \mathscr{E}^{\mathrm{i}}\left(y^{\mathrm{F}}\right), v_{h}\right\rangle+\left\langle\delta \mathscr{E}^{\mathrm{c}}\left(y^{\mathrm{F}}\right), v_{h}\right\rangle, \quad \forall v_{h} \in \mathscr{U}_{h} .
$$


To simplify the notation, we drop the $y^{\mathrm{F}}$ dependence from the following expressions in this section, for example, we write $\mathscr{E}$ a instead of $\mathscr{E}^{\mathrm{a}}\left(y^{\mathrm{F}}\right), \nabla_{\rho} V$ instead of $\nabla_{\rho} V\left(D y^{\mathrm{F}}\right)$, and so forth. Here, $\nabla_{\rho} V$ denotes the partial derivative of $V$ with respect to the $D_{\rho} y$ component. Since $\nabla_{\rho} V=-\nabla_{-\rho} V$, we only consider half of the directions in the interaction range: fix $\mathcal{R}^{+} \subset \mathcal{R}$ such that $\mathcal{R}^{+} \cup\left(-\mathcal{R}^{+}\right)=\mathcal{R}$ and $\mathcal{R}^{+} \cap\left(-\mathcal{R}^{+}\right)=\varnothing$.

As proposed in [29], a necessary and sufficient condition on the reconstruction parameters $C_{\ell}$ to satisfy the force patch test (3.4) for all $V \in C^{\infty}\left(\left(\mathbb{R}^{d}\right)^{\mathcal{R}}\right)$ is

$$
c_{\rho}^{\mathrm{a}}(\ell)+c_{\rho}^{\mathrm{i}}(\ell)+c_{\rho}^{\mathrm{c}}(\ell)=0,
$$

for $\ell \in \Lambda^{\mathrm{i}}+\mathcal{R}$, and $\rho \in \mathcal{R}^{+}$. The coefficients $c_{\rho}^{\mathrm{a}}(\ell), c_{\rho}^{\mathrm{i}}(\ell)$ and $c_{\rho}^{\mathrm{c}}(\ell)$ are geometric parameters with respect to the underlying lattice and the interface geometry, formulated by collecting all the coefficients for the terms $\nabla_{\rho} V$. of the first variations of a/c coupling energy as in the following equations (3.7). The interface coefficients $c_{\rho}^{i}(\ell)$ depend linearly on the unknown reconstruction parameters $C_{\ell ; \rho, \zeta}$. Then, by (3.5), we have

$$
\begin{aligned}
\left\langle\delta \mathscr{E}^{\mathrm{a}}, v_{h}\right\rangle= & \sum_{\ell \in \Lambda^{\mathrm{a}}+\mathcal{R} \rho \in \mathcal{R}^{+}} c_{\rho}^{\mathrm{a}}(\ell)\left[\nabla_{\rho} V \cdot v_{h}(\ell)\right] \\
= & \sum_{\substack{\rho \in \mathcal{R}^{+} \\
\ell \in \Lambda^{\mathrm{a}}-\rho}}\left[\nabla_{\rho} V \cdot v_{h}(\ell)\right]-\sum_{\substack{\rho \in \mathcal{R}^{+} \\
\ell \in \Lambda^{\mathrm{a}}+\rho}}\left[\nabla_{\rho} V \cdot v_{h}(\ell)\right], \\
\left\langle\delta \mathscr{E}^{\mathrm{i}}, v_{h}\right\rangle= & \sum_{\ell \in \Lambda^{i}+\mathcal{R} \rho \in \mathcal{R}^{+}} c_{\rho}^{\mathrm{i}}(\ell)\left[\nabla_{\rho} V \cdot v_{h}(\ell)\right] \\
= & \sum_{\substack{\zeta \in \mathcal{R} \\
\ell \in \Lambda^{\mathrm{i}}+\varsigma}} \omega_{\ell-\varsigma}^{\mathrm{i}} \sum_{\rho \in \mathcal{R}^{+}}\left(C_{\ell-\varsigma ; \rho, \zeta}-C_{\ell-\varsigma ;-\rho, \zeta}\right)\left[\nabla_{\rho} V \cdot v_{h}(\ell)\right] \\
& -\sum_{\ell \in \Lambda^{\mathrm{i}}} \omega_{\ell}^{\mathrm{i}} \sum_{\rho \in \mathcal{R}^{+}} \sum_{\zeta \in \mathcal{R}}\left(C_{\ell ; \rho, \zeta}-C_{\ell ;-\rho, \zeta}\right)\left[\nabla_{\rho} V \cdot v_{h}(\ell)\right], \\
\left\langle\delta \mathscr{E} \mathrm{c}, v_{h}\right\rangle= & \sum_{T} \sum_{\rho \in \mathcal{R}^{+}} \sum_{i=1}^{3} 2 \frac{\omega_{T}}{\operatorname{det} \mathrm{A}} \nabla_{T} \phi_{i}^{T} \cdot \rho\left[\nabla_{\rho} V \cdot v_{h, i}^{T}\right], \\
= & \sum_{T} \sum_{\rho \in \mathcal{R}^{+}} \sum_{i=1}^{3} 2 \frac{\omega_{T}}{\operatorname{det} \mathrm{A}} \nabla_{T} \phi_{i}^{T} \cdot \rho\left[\nabla_{\rho} V \cdot v_{h, i}^{T}\right],
\end{aligned}
$$

where the nodes $\ell_{i}^{T}$ are the three corners of the triangle $T, v_{h, i}^{T}=v\left(\ell_{i}^{T}\right)$ and $\phi_{i}^{T}$ are the three nodal linear bases corresponding to $v_{h, i}^{T}, i=1,2,3$.

The force patch test is automatically satisfied for the atomistic model and the CauchyBorn continuum model.Therefore, we only need to consider the force consistency for those sites with the extended interface region $\Lambda^{\mathrm{i}}+\mathcal{R}:=\left\{\ell \in \Lambda \mid \exists \ell^{\prime} \in \Lambda^{\mathrm{i}}, \exists \rho \in \mathcal{R}\right.$, such that $\ell=$ $\left.\ell^{\prime}+\rho\right\}$. Therefore (3.6) for $\ell \in \Lambda^{\mathrm{i}}+\mathcal{R}$ together with (3.3) for $\ell \in \Lambda^{\mathrm{i}}$ form a linear system for the unknown parameters $C_{\ell ; \rho, \zeta}$. 


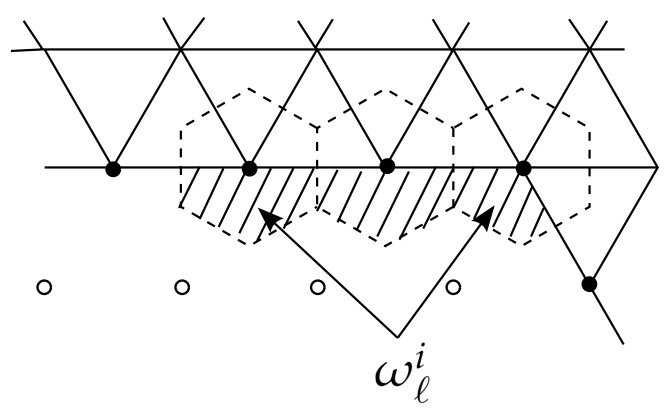

Figure 2: Effective Voronoi cells for the interface nodes (filled circles) are the shaded area in the above figure. $\omega_{\ell}^{\mathrm{i}}<1$ for the outmost interface atoms which are adjacent to the continuum region.

Remark 3.1. In $[28,29]$, we choose $\omega_{\ell}^{i}=\operatorname{vor}(\ell), \forall \ell \in \Lambda^{i}$. To reduce the degrees of freedom of the reconstruction parameters and also the number of constraint equations, we can use a variation of the local reflection method in [29]. Namely, we choose $\omega_{\ell}^{i}=\operatorname{vor}(\ell) \cap \Omega^{\mathrm{a}}$, and enforce that $C_{\ell ; \rho, \zeta}=0$ for $\ell \in \Lambda^{\mathrm{i}}$ and $\ell+\varsigma \in \Omega^{\mathrm{c}}$, then we only need to impose the force balance equation for $\left(\Lambda^{\mathrm{i}}+\mathcal{R}\right) \cap \Lambda^{\mathrm{a}}$. It has been shown that in [29], the linear system for the reconstruction parameters is underdetermined, and up to numerical accuracy, the solution exists and therefore it is not unique.

\subsection{Consistency and optimisation of reconstruction parameters}

Due to the non-uniqueness of the reconstruction parameters $C_{\ell, \rho, \xi}$, we need to choose the "optimal" parameters. A naive idea is to use a least-squares approach to minimize $\|C\|_{\ell^{2}}$,

$$
\text { minimize } \sum_{\ell \in \Lambda^{\mathrm{i}} \rho, \zeta \in \mathcal{R}(\ell)}\left|C_{\ell ; \rho, \varsigma}\right|^{2} \quad \text { subject to (3.3) and (3.6). }
$$

However, the resulting parameters do not give a convergent method.

It is shown in [21, Thm. 6.1], under the assumptions that $d=2$ and that the atomistic region $\Omega^{\mathrm{a}}$ is connected, any a/c coupling scheme of the type (2.4) satisfying the energy and force patch tests (3.2), (3.4) is first-order consistent: if $y=y^{\mathrm{B}}$ in $\Lambda \backslash \Omega_{R}$ and if $\tilde{y}$ is an $H_{\text {loc }}^{2}$-conforming interpolant of $y$, then

$$
\left\langle\delta \mathscr{E}(y)-\delta \mathscr{E}^{\mathfrak{h}}\left(I_{h} y\right), v_{h}\right\rangle \leq C_{1}\left\|h \nabla^{2} \tilde{y}\right\|_{L^{2}\left(\tilde{\Omega}^{c}\right)}\left\|\nabla v_{h}\right\|_{L^{2}}
$$

where $C_{1}$ is independent of $y$. The dependence of $C_{1}$ on the reconstruction parameters $C_{\ell}$ is analyzed in [29],

$$
\begin{gathered}
C_{1} \leq C_{1}^{\prime}\left(1+\operatorname{width}\left(\Lambda^{\mathrm{i}}\right)\right) \sum_{\rho, \zeta \in \mathcal{R}}|\rho||\zeta| M_{\rho, \zeta}+C_{1}^{\prime \prime}, \\
\text { where } \quad M_{\rho, \zeta}=\max _{\ell \in \Lambda^{\mathrm{i}}} \sum_{\tau, \tau^{\prime} \in \mathcal{R}(\ell)}\left|V_{\tau, \tau^{\prime}}\left(C_{\ell} \cdot D y(\ell)\right)\right|\left|C_{\ell ; \tau, \rho}\right|\left|C_{\ell ; \tau^{\prime}, \zeta}\right| .
\end{gathered}
$$


$C_{1}^{\prime}$ is a generic constant and $C_{1}^{\prime \prime}$ does not depend on the reconstruction parameters.

Intuitively one may think of

$$
M(\ell):=\sum_{\rho, \zeta}|\rho||\varsigma| \sum_{\tau, \tau^{\prime}}\left|V_{\tau, \tau^{\prime}}\left(C_{\ell} \cdot D y(\ell)\right)\right|\left|C_{\ell ; \tau, \rho}\right|\left|C_{\ell ; \tau^{\prime}, \varsigma}\right|
$$

to be a realistic ( $\ell$-dependent) pre-factor. With generic structural assumption $\mid V_{\tau, \tau^{\prime}}\left(C_{\ell}\right.$. $D y(\ell)) \mid \lesssim \omega(|\tau|) \omega\left(\left|\tau^{\prime}\right|\right)$, where $\omega$ has some decay that is determined by the specific interaction potential, see the discussion for an EAM type potential in Appendix B.2 [26]. We obtain that

$$
\begin{aligned}
M(\ell) & \lesssim \sum_{\rho, \zeta}|\rho||\zeta| \sum_{\tau, \tau^{\prime}} \omega(|\tau|) \omega\left(\left|\tau^{\prime}\right|\right)\left|C_{\ell ; \tau, \rho}\right|\left|C_{\ell ; \tau^{\prime}, \zeta}\right| \\
& =\left(\sum_{\rho, \tau}|\rho| \omega(|\tau|)\left|C_{\ell ; \tau, \rho}\right|\right)\left(\sum_{\zeta, \tau^{\prime}}|\zeta| \omega\left(\left|\tau^{\prime}\right|\right)\left|C_{\ell ; \tau^{\prime}, \zeta}\right|\right) \\
& =\left(\sum_{\rho, \tau}|\rho| \omega(|\tau|)\left|C_{\ell ; \tau, \rho}\right|\right)^{2} .
\end{aligned}
$$

This indicates that, instead of $\|C\|_{\ell^{2}}$, we should minimize $\max _{\ell \in \Lambda^{\mathrm{i}}} \sum_{\rho, \tau}|\rho| \omega(|\tau|)\left|C_{\ell ; \tau, \rho}\right|$. Since we do not in general know the generic weights $\omega$, we simply drop them, and instead minimize $\sum_{\rho, \tau}\left|C_{\ell ; \tau, \rho}\right|$. Further, taking the maximum of $\ell \in \Lambda^{\mathrm{i}}$ leads to a difficult and computationally expensive multi-objective optimisation problem. Instead, we propose to minimize the $\ell^{1}$-norm of all the coefficients:

$$
\text { minimise } \sum_{\ell \in \Lambda^{\mathrm{i}} \rho, \zeta \in \mathcal{R}(\ell)}\left|C_{\ell ; \rho, \varsigma}\right| \quad \text { subject to (3.3) and (3.6). }
$$

We remark that with the simplifications, the reconstruction parameters only depend on the interaction range, not the particular form of interaction potentials, also, $\ell^{1}$ minimization tends to generate "sparse" reconstruction parameters which may present some gain in computational cost in the energy and force assembly routines for $E^{\text {ac }}$.

\subsection{Stability and stabilisation}

The stability of a / c coupling method is of great importance for numerical analysis and the issue of critical stability is essential for practitioners. The issue of stability and (in)stability of a/c coupling scheme is discussed in detail in [24], in particular, a stabilization mechanism is proposed to reduce the stability gap between the a/c coupling methods and the corresponding atomistic model (ground truth). We sketch those results in this subsection.

We expect a/c coupling method has the following stability estimate of the form

$$
\left\langle\delta^{2} \mathscr{E}^{\mathrm{h}}\left(I_{h} y\right) v_{h}, v_{h}\right\rangle \geq c_{0}\left\|\nabla v_{h}\right\|_{L^{2}}^{2},
$$

together with consistency and other technical assumptions, we can prove the convergence of the a/c coupling method with inverse function theorem with the form $\left\|u-u_{h}\right\| \lesssim$ consistency error $/ c_{0}$. 
Also, we have the observation that for any uniform deformation, the stability constant of a/c coupling method is less or equal to the stability constant of the corresponding atomistic model. This motivate the introduction of the following stabilization of the interface potential

$$
V_{\ell}^{\mathrm{i}}\left(y_{h}\right):=V\left(C_{\ell} \cdot D y_{h}(\ell)\right)+\kappa\left|D_{\mathrm{nn}}^{2} y_{h}(\ell)\right|^{2},
$$

where $\kappa \geq 0$ is a stabilisation parameter, and $\left|D_{\text {nn }}^{2} y_{h}(\ell)\right|^{2}$ is defined as follows: we choose $m \geq d$ linearly independent "nearest-neighbor" directions $a_{1}, \cdots, a_{m}$ in the lattice, and denote

$$
\left|D_{\mathrm{nn}}^{2} y_{h}(\ell)\right|^{2}:=\sum_{j=1}^{m}\left|y_{h}\left(\ell+a_{j}\right)-2 y_{h}(\ell)+y_{h}\left(\ell-a_{j}\right)\right|^{2}
$$

If $\kappa$ is $\mathcal{O}(1)$ constant, the stabilization term does not affect consistency, the reconstruction parameters $C_{\ell}$ are still determined by (3.11). However, the stabilisation will affect the computation of the stress tensor in the interface region, which we will introduce in the following section. In [24], we have theoretically proved for some symmetric configuration and numerical justified for prototypical examples that such stabilization can reduce the stability gap and suppress spurious critical mode for the a/c coupling methods.

\section{Stress formulation}

The stress formulation plays a significant role in the analysis of a/c coupling methods. For a general energy functional $\mathscr{E}(y)$ defined on the deformed configuration $y-y^{B} \in \mathscr{U}^{1,2}$, we can define $\sigma(y)$ in the following, which is an analogue of the first Piola-Kirchhoff stress

$$
\langle\delta \mathscr{E}(y), v\rangle=\int_{\mathbb{R}^{d}} \sigma(y): \nabla v \mathrm{~d} x
$$

for any $v \in \mathscr{U}^{1,2}$.

In particular, from the first variation of the a/c energy functional (3.5) and (3.7), we expect to generalize the above stress formulation to atomistic, continuum and the a/c energy functionals, namely, for any $v_{h} \in \mathscr{U}_{h}$,

$$
\begin{aligned}
\left\langle\delta \mathscr{E}^{\mathrm{h}}\left(y_{h}\right), v_{h}\right\rangle & =\left\langle\delta \mathscr{E}^{\mathrm{a}}, v_{h}\right\rangle+\left\langle\delta \mathscr{E}^{\mathrm{i}}, v_{h}\right\rangle+\left\langle\delta \mathscr{E}^{\mathrm{c}}, v_{h}\right\rangle \\
& =\int_{\Omega^{\mathrm{a}}} \sigma^{\mathrm{a}}: \nabla v_{h} \mathrm{~d} x+\int_{\Omega^{\mathrm{i}}} \sigma^{\mathrm{i}}: \nabla v_{h} \mathrm{~d} x+\int_{\Omega^{\mathrm{c}}} \sigma^{\mathrm{c}}: \nabla_{T} v_{h} \\
& =\int_{\Omega} \sigma^{\mathrm{h}}: \nabla v_{h} \mathrm{~d} x .
\end{aligned}
$$

The expressions of $\sigma^{\mathrm{a}}, \sigma^{\mathrm{i}}$ and $\sigma^{\mathrm{c}}$ are in (4.13) to (4.15).

In this section, we first review the stress tensor formula derived by [26] in Section 4.1 which is essential for the a priori analysis, however, this formula is not suitable for the a posteriori estimate. In Section 4.2 we introduce a novel computable stress tensor expression, which is convenient for the purpose of a posterior error estimation and adaptive algorithm. We discuss the assembly of stress tensor for our model problem in Section A.1. 


\subsection{Stress tensor formulation in [26]}

We first discuss the formula for $\sigma^{\text {a }}$. For simplicity, consider full atomistic energy $\mathscr{E}$ in (2.2). The "canonical weak form" of $\delta \mathscr{E}$ is

$$
\langle\delta \mathscr{E}(u), v\rangle=\sum_{\ell \in \Lambda} \sum_{\rho \in \mathcal{R}} V_{\ell, \rho}(u) \cdot D_{\rho} v(\ell), \quad \text { for } v \in \mathscr{U}^{1,2} .
$$

Now we define a modified version of the canonical weak form. Let

$$
v^{*}:=\bar{\zeta} * \bar{v} .
$$

The finite differences $D_{\rho} v^{*}(\zeta)$ can be expressed as in [35],

$$
\begin{aligned}
D_{\rho} v^{*}(\ell) & =\int_{s=0}^{1} \nabla_{\rho} v^{*}(\ell+s \rho) \mathrm{d} s=\int_{\mathbb{R}^{d}} \int_{s=0}^{1} \bar{\zeta}(\ell+s \rho-x) \nabla_{\rho} \bar{v}(x) \mathrm{d} s \mathrm{~d} x \\
& =\int_{\mathbb{R}^{d}} \chi_{\ell, \rho}(x) \nabla_{\rho} \bar{v}(x) \mathrm{d} x,
\end{aligned}
$$

where $\chi_{\ell, \rho}$ is a generic weighting function defined as below, can be understood as a mollified version of the line measure

$$
\chi_{\ell, \rho}(x):=\int_{0}^{1} \bar{\zeta}(\ell+t \rho-x) \mathrm{d} t .
$$

Now, we replace the test function in (4.1) from $v$ to $v^{*}$,

$$
\begin{aligned}
\left\langle\delta \mathscr{E}(y), v^{*}\right\rangle & =\sum_{\ell \in \Lambda^{a}} \sum_{\rho \in \mathcal{R}} \partial_{\rho} V_{\ell} \cdot \int_{\mathbb{R}^{d}} \chi_{\ell, \rho}(x) \nabla_{\rho} \bar{v}(x) \mathrm{d} x \\
& =\int_{\mathbb{R}^{d}}\left\{\sum_{\ell \in \Lambda^{a}} \sum_{\rho \in \mathcal{R}}\left[\partial_{\rho} V_{\ell} \otimes \rho\right] \chi_{\ell, \rho}(x)\right\}: \nabla \bar{v} \mathrm{~d} x .
\end{aligned}
$$

Thus, we have shown that for $y-y^{\mathrm{B}} \in \mathscr{U}^{1,2}$ and $v \in \mathscr{U}$ with compact support,

$$
\left\langle\delta \mathscr{E}(y), v^{*}\right\rangle=\int_{\mathbb{R}^{d}} \Sigma^{\mathrm{a}}(y ; x): \nabla \bar{v} \mathrm{~d} x,
$$

with

$$
\Sigma^{\mathrm{a}}(y ; x):=\sum_{\ell \in \Lambda^{\mathrm{a}}} \sum_{\rho \in \mathcal{R}}\left[\partial_{\rho} V_{\ell} \otimes \rho\right] \chi_{\ell, \rho}(x) .
$$

Through an analogy to the analysis above, $\left\langle\delta \mathscr{E}^{\mathrm{i}}(y), v^{*}\right\rangle$ could be written as

$$
\left\langle\delta \mathscr{E}^{\mathrm{i}}(y), v^{*}\right\rangle=\int_{\mathbb{R}^{d}} \Sigma^{\mathrm{i}}(y ; x): \nabla \bar{v} \mathrm{~d} x
$$

where

$$
\Sigma^{\mathrm{i}}(y ; x):=\sum_{\ell \in \Lambda^{\mathrm{i}}} \sum_{\rho \in \mathcal{R}}\left[\omega_{\ell}^{\mathrm{i}} \partial_{\rho} V_{\ell}^{\mathrm{i}} \otimes \rho\right] \chi_{\ell, \rho}(x)
$$


Finally, the first Piola-Kirchhoff stress of the Cauchy-Born model gives

$$
\Sigma^{\mathrm{C}}(y ; x)=\partial W(\nabla y(x))
$$

By proper regularity assumptions on $V$ and $y$, it was shown in [26, Theorem 4.3] that $\Sigma^{\mathrm{C}}(y ; x)$ is second order consistent to $\Sigma^{\mathrm{a}}(y ; x)$.

The stress tensor expression introduced in this section is important for the a priori analysis of Caucy-Born continuum model in [26] and blended a/c coupling method [13]. However, since $\Sigma^{\mathrm{a}}(y ; x)$ and $\Sigma^{\mathrm{i}}(y ; x)$ depend on $x$ through $\chi_{\ell, \rho}(x)$, it is relatively difficult to calculate the value of them, which is crucial for the a posteriori error estimates and adaptive algorithm. In the next section, we will introduce a computable expression of stress tensor.

\subsection{A computable stress tensor formulation}

For the nearest neighbor interactions [41], we use the canonical expressions of $\delta \mathscr{E}\left(\delta \mathscr{E}^{\mathrm{h}}\right)$ to define $\sigma^{\mathrm{a}}\left(\sigma^{\mathrm{h}}\right)$. In that case, $\sigma^{\mathrm{a}}\left(\sigma^{\mathrm{h}}\right)$ is piecewise constant over the triangulation $\mathcal{T}_{\mathrm{a}}\left(\mathcal{T}_{\mathrm{h}}\right)$. In this section, we will extend this formulation to general finite range interactions.

Consider the canonical weak form of $\delta \mathscr{E}$ in (4.1), instead of replacing $v$ with $v^{*}$, we distribute $D_{\rho} v$ to relevant triangles in order to transfer the sum with respect to atoms to the sum over elements in the micro-triangulation $\mathcal{T}_{\mathrm{a}}$ induced by the reference lattice $\Lambda$. We express $D_{\rho} v$ as

$$
D_{\rho} v(\ell)=\sum_{T \in \mathcal{T}_{\ell}^{\rho}} \omega_{\ell}^{\rho}(T) \nabla_{T} v \cdot \rho,
$$

where $\mathcal{T}_{\ell}^{\rho}:=\left\{T \in \mathcal{T}_{\mathrm{a}} \mid\right.$ length $\left.(T \cap(\ell, \ell+\rho))>0\right\}$ is the set which contains the elements that form the compact support of bond $(\ell, \ell+\rho), \omega_{\ell}^{\rho}(T)$ is an appropriate weight function. The implementation details for two dimensional triangular lattice will be discussed in detail in Section A.1.

$$
\begin{aligned}
& \text { For any } y-y^{\mathrm{B}} \in \mathscr{U}^{1,2}, v \in \mathscr{U}^{1,2}, \\
& \qquad \begin{aligned}
\langle\delta \mathscr{E}(y), v\rangle & =\sum_{\ell \in \Lambda} \sum_{\rho \in \mathcal{R}_{\ell}} \partial_{\rho} V_{\ell} \cdot D_{\rho} v \\
& =\sum_{\ell \in \Lambda} \sum_{\rho \in \mathcal{R}_{\ell}} \partial_{\rho} V_{\ell} \cdot\left(\omega_{\rho}\left(\sum_{b=(\ell, \ell+\rho) \cap T \neq \varnothing} \nabla_{T} v\right) \cdot \rho\right) \\
& =\sum_{\ell \in \Lambda} \sum_{\rho \in \mathcal{R}_{\ell}} \frac{2|T|}{\operatorname{det} \mathrm{A}} \partial_{\rho} V_{\ell} \otimes \rho:\left(\omega_{\rho} \sum_{b=(\ell, \ell+\rho) \cap T \neq \varnothing} \nabla_{T} v\right) \\
& =|T| \sum_{T \in \mathcal{T}_{\mathrm{a}} \rho \in \mathcal{R}_{\ell}} \frac{1}{\operatorname{det} \mathrm{A}_{b=(\ell, \ell+\rho) \cap T \neq \varnothing}} \sum_{\omega_{\rho} \partial_{\rho} V_{\ell} \otimes \rho:\left(\nabla_{T} v\right), \forall v \in \mathscr{U},}
\end{aligned}
\end{aligned}
$$


where $\omega_{\ell}^{\rho}(T)$ represents the contribution of the value of $\partial_{\rho} V\left(\nabla_{T} y_{h}\right) \otimes \rho$ from element $T$, which depends on the specific type of $\rho \in \mathcal{R}$.

Recall from (2.4) and (3.1), the GRAC a/c coupling energy functional is of the form

$$
\mathscr{E}^{\mathrm{h}}\left(y_{h}\right):=\sum_{\ell \in \Lambda^{\mathrm{a}}} V_{\ell}\left(y_{h}\right)+\sum_{\ell \in \Lambda^{\mathrm{i}}} \omega_{\ell} V_{\ell}^{\mathrm{i}}\left(y_{h}\right)+\sum_{T \in \mathcal{T}_{\mathrm{h}}} \omega_{T} W\left(\left.\nabla y_{h}\right|_{T}\right),
$$

with

$$
V_{\ell}^{\mathrm{i}}(y):=V_{\ell}\left(\left(\sum_{\zeta \in \mathcal{R}_{\ell}} C_{\ell ; \rho, \zeta} D_{\zeta} y(\ell)\right)_{\rho \in \mathcal{R}_{\ell}}\right) .
$$

With the decomposition of the first variation of GRAC in (3.5), the first variation of the interface energy $\mathscr{E}^{\mathrm{i}}$ has the following form

$$
\begin{aligned}
\left\langle\delta \sum_{\ell \in \Lambda^{\mathrm{i}}} \omega_{\ell} V_{\ell}^{\mathrm{i}}\left(y_{h}\right), v_{h}\right\rangle & =\left\langle\delta \sum_{\ell \in \Lambda^{\mathrm{i}}} \omega_{\ell} V_{\ell}\left(\left(\sum_{\zeta \in \mathcal{R}_{\ell}} C_{\ell ; \rho, \zeta} D_{\zeta} y_{h}\right)_{\rho \in \mathcal{R}_{\ell}}\right), v_{h}\right\rangle \\
& =\sum_{\ell \in \Lambda^{\mathrm{i}}} \omega_{\ell} \sum_{\rho \in \mathcal{R}_{\ell}} \partial_{\rho} V_{\ell} \cdot\left(\sum_{\zeta \in \mathcal{R}_{\ell}} C_{\ell ; \rho, \zeta} D_{\zeta} v_{h}(\ell)\right) \\
& =\sum_{\ell \in \Lambda^{\mathrm{i}}} \omega_{\ell} \sum_{\rho \in \mathcal{R}_{\ell} \in \mathcal{R}_{\ell}} \partial_{\rho} V_{\ell} C_{\ell ; \rho, \zeta} \cdot\left(\omega_{\zeta}\left(\sum_{b=(\ell, \ell+\zeta) \cap T \neq \varnothing} \nabla_{T} v_{h}\right) \cdot \varsigma\right) \\
& =\sum_{\ell \in \Lambda^{\mathrm{i}}} \frac{2 \omega_{\ell}|T|}{\operatorname{det} \mathrm{A}} \sum_{\zeta \in \mathcal{R}_{\ell}}\left(\sum_{\rho \in \mathcal{R}_{\ell}} C_{\ell ; \rho, \zeta} \partial_{\rho} V_{\ell} \otimes \zeta\right):\left(\omega_{\zeta} \sum_{b=(\ell, \ell+\zeta) \cap T \neq \varnothing} \nabla_{T} v_{h}\right) \\
& =\sum_{T \in \mathcal{T}_{\mathrm{h}}^{\mathrm{i}}} \frac{|T|}{\operatorname{det} \mathrm{A}} \sum_{\zeta \in \mathcal{R}_{\ell} b=(\ell, \ell+\varsigma) \cap T \neq \varnothing} \sum_{\rho \in \mathcal{R}_{\ell}} 2 \omega_{\ell, \rho, \zeta}\left(\sum_{\zeta} \partial_{\rho} V_{\ell} \otimes \zeta\right):\left(\nabla_{T} v_{h}\right) .
\end{aligned}
$$

Consider the continuum model, for any $y_{h}-y^{\mathrm{B}} \in \mathscr{U}_{h}, v_{h} \in \mathscr{U}_{h}$, we have

$$
\begin{aligned}
\left\langle\delta \mathscr{E}^{\mathscr{C}}\left(y_{h}\right), v_{h}\right\rangle & =\sum_{T \in \mathcal{T}_{\mathrm{h}}}|T| \partial W\left(\nabla_{T} y_{h}\right) \\
& =\sum_{T \in \mathcal{T}_{\mathrm{h}}}|T| \sum_{\rho \in \mathcal{R}} \partial_{\rho} V\left(\nabla_{T} y_{h}\right) \otimes \rho: \nabla_{T} v_{h} .
\end{aligned}
$$

Adding the stabilization term as in (3.13), we are ready to obtain the first variation of the (stabilized) GRAC coupling method

$$
\begin{aligned}
\left\langle\delta \mathscr{E}^{\mathrm{eh}}\left(y_{h}\right), v_{h}\right\rangle= & \sum_{T \in \mathcal{T}_{\mathrm{h}}^{\mathrm{a}} \cup \mathcal{T}_{\mathrm{h}}^{\mathrm{i}}} \frac{|T|}{\operatorname{det} \mathrm{A}} \sum_{\rho \in \mathcal{R}_{\ell} b=(\ell, \ell+\rho) \cap T \neq \varnothing} \sum_{T \in \mathcal{T}_{\mathrm{h}}} 2 \omega_{\ell} \omega_{\ell}^{\rho}(T) \partial_{\rho} V_{\ell}^{h} \otimes \rho:\left(\nabla_{T} v_{h}\right) \\
& +\sum_{T} \frac{\omega_{T}}{\operatorname{det} \mathrm{A}} \sum_{\rho \in \mathcal{R}} \partial_{\rho} V\left(\nabla_{T} y_{h}\right) \otimes \rho: \nabla_{T} v_{h}, \\
& +C_{\text {stab }} \sum_{T^{\prime} \in \mathcal{T}_{\mathrm{h}}^{i}} \sum_{\zeta \in \mathbb{R}_{\mathrm{nn}} b^{\prime}=(\ell, \ell+\zeta) \cap T^{\prime} \neq \varnothing} 2 \omega_{\ell}^{\zeta}(T) \mathrm{d}_{\mathrm{nn}}^{3}(\ell, \zeta) \otimes \zeta: \nabla_{T^{\prime}} v_{h} \in \mathscr{U}_{h},
\end{aligned}
$$


where

$$
\begin{aligned}
& \partial_{\rho} V_{\ell}^{h}:= \begin{cases}\partial_{\rho} V_{\ell}, & \ell \in \Lambda^{a}, \\
\sum_{\varsigma \in \mathcal{R}_{\ell}} C_{\ell ; \zeta, \rho} \partial_{\varsigma} V_{\ell}, & \ell \in \Lambda^{i},\end{cases} \\
& \mathrm{d}_{\mathrm{nn}}^{3}(\ell, \zeta)=-y_{h}(\ell+2 \zeta)+3 y_{h}(\ell+\zeta)-3 y_{h}(\ell)+y_{h}(\ell-\zeta),
\end{aligned}
$$

and $C_{\text {stab }}$ is a constant equal to 1 if stabilisation is applied, and 0 otherwise.

We can define the atomistic stress tensor $\sigma^{\text {a }}$ (with respect to micro-triangulation $\mathcal{T}_{\mathrm{a}}$ ), the continuum stress tensor $\sigma^{\mathrm{c}}$, and the a/c stress tensor $\sigma^{\mathrm{h}}$ by the following first variations of different models

$$
\begin{aligned}
& \left\langle\delta \mathscr{E}^{\mathrm{a}}(y), v\right\rangle=\sum_{T \in \mathcal{T}_{\mathrm{a}}}|T| \sigma^{\mathrm{a}}(y ; T): \nabla_{T} v, \forall v \in \mathscr{U}, \\
& \left\langle\delta \mathscr{E}^{\mathrm{c}}\left(y_{h}\right), v_{h}\right\rangle=\sum_{T \in \mathcal{T}_{\mathrm{h}}}|T| \sigma^{\mathrm{c}}\left(y_{h} ; T\right): \nabla_{T} v_{h}, \forall v_{h} \in \mathscr{U}_{h}, \\
& \left\langle\delta \mathscr{E}^{\mathrm{h}}\left(y_{h}\right), v_{h}\right\rangle=\sum_{T \in \mathcal{T}_{\mathrm{h}}}|T| \sigma^{\mathrm{h}}\left(y_{h} ; T\right): \nabla_{T} v_{h}, \forall v_{h} \in \mathscr{U}_{h} .
\end{aligned}
$$

From the above discussions, we have the following "canonical" choices for $\sigma^{\mathrm{a}}, \sigma^{\mathrm{c}}$ and $\sigma^{\mathrm{h}}$

$$
\begin{aligned}
& \sigma^{\mathrm{a}}(y ; T):=\frac{1}{\operatorname{det} \mathrm{A}} \sum_{\rho \in \mathcal{R}_{\ell} b=(\ell, \ell+\rho) \cap T \neq \varnothing} \sum_{\ell} 2 \omega_{\ell}^{\rho}(T) \partial_{\rho} V_{\ell} \otimes \rho, \\
& \sigma^{\mathcal{c}}\left(y_{h} ; T\right):=\frac{1}{\operatorname{det} A} \sum_{\rho \in \mathcal{R}} \partial_{\rho} V\left(\nabla_{T} y_{h}\right) \otimes \rho, \\
& \sigma^{\mathrm{h}}\left(y_{h} ; T\right):=\frac{1}{\operatorname{det} \mathrm{A}}\left(\sum_{\rho \in \mathcal{R}_{\ell} b=(\ell, \ell+\rho) \cap T \neq \varnothing} \sum 2 \omega_{\ell} \omega_{\ell}^{\rho}(T) \partial_{\rho} V_{\ell}^{h} \otimes \rho+\frac{\omega_{T}}{|T|} \sigma^{\mathrm{c}}\left(y_{h} ; T\right)\right. \\
& \left.+C_{\text {stab }} \sum_{\zeta \in \mathbb{R}_{\mathrm{nn}} b^{\prime}=(\ell, \ell+\zeta) \cap T^{\prime} \neq \varnothing} 2 \omega_{\ell}^{\zeta}\left(T^{\prime}\right) \mathrm{d}_{\mathrm{nn}}^{3}(\ell, \zeta) \otimes \zeta\right) .
\end{aligned}
$$

Remark 4.1. Notice that the proposed stress tensor formulation is the same as that in [21, Proposition 13] which is used for the a priori analysis and not for the actual computation. In this paper, we use the formulation to construct a computable stress tensor and compute the a posteriori error estimators. The bond density lemma in [21,34] might be useful to simplify the computation of the stress tensor.

However, the stress tensors defined through (4.13)-(4.15) are not unique due to the following results.

Definition 4.1. We call piecewise constant tensor field $\sigma \in \mathrm{P}_{0}(\mathcal{T})^{2 \times 2}$ divergence free if

$$
\sum_{T \in \mathcal{T}}|T| \sigma(T): \nabla_{T} v \equiv 0, \forall v \in\left(\mathrm{P}_{1}(\mathcal{T})\right)^{2}
$$


Corollary 4.1. By definitions (4.15), it is easy to know that the force patch test condition (3.4) is equivalent to that $\sigma^{h}\left(y_{F}\right)$ is divergence free for any constant deformation gradient $F$.

The discrete divergence free tensor fields over the triangulation $\mathcal{T}$ can be characterized by the non-conforming Crouzeix-Raviart finite elements [21,28]. The CrouzeixRaviart finite element space over $\mathcal{T}$ is defined as

$$
\begin{aligned}
N_{1}(\mathcal{T})=\left\{c: \bigcup_{T \in \mathcal{T}} \operatorname{int}(T) \rightarrow \mathbb{R} \mid\right. & c \text { is piecewise affine w.r.t. } \mathcal{T}, \text { and } \\
& \text { continuous in edge midpoints } \left.q_{f}, \forall f \in \mathcal{F}\right\} .
\end{aligned}
$$

The following lemma in [28] characterizes the discrete divergence-free tensor field.

Lemma 4.1. A tensor field $\sigma \in P_{0}(\mathcal{T})^{2 \times 2}$ is divergence free if and only if there exists a constant $\sigma_{0} \in \mathbb{R}^{2 \times 2}$ and a function $c \in N_{1}(\mathcal{T})^{2}$ such that

$$
\sigma=\sigma_{0}+\nabla c J, \quad \text { where } J=\left[\begin{array}{cc}
0 & -1 \\
1 & 0
\end{array}\right] \in S O(2)
$$

The following corollary provides a representation of the stress tensors defined in (4.13)-(4.15).

Corollary 4.2. The stress tensors in the definitions (4.13)-(4.15) are not unique. Given any stress tensor $\sigma \in P_{0}(\mathcal{T})^{2 \times 2}$ satisfies one of the definitions (4.13)-(4.15), where $\mathcal{T}$ is the corresponding triangulation. Define the admissible set as $\operatorname{Adm}(\sigma):=\left\{\sigma+\nabla c J, c \in N_{1}(\mathcal{T})^{2}\right\}$, then any $\sigma^{\prime} \in$ $\operatorname{Adm}(\sigma)$ satisfies the definition of stress tensor.

\section{Adaptive algorithms and numerical experiments}

We have derived the following a posteriori estimate in [41, Theorem 3.1]: let $y_{h}$ be the a/c solution, for any $v \in \mathscr{U}^{1,2}$, the residual $\mathrm{R}[v]=\left\langle\delta \mathscr{E}\left(I_{\mathrm{a}} y_{h}\right), v\right\rangle$ is bounded by the sum of the following estimators

$$
\left\langle\delta \mathscr{E}\left(I_{\mathrm{a}} y_{h}\right), v\right\rangle \leq\left(\eta_{T}\left(y_{h}\right)+\eta_{M}\left(y_{h}\right)+\eta_{C}\left(y_{h}\right)\right)\|\nabla v\|_{L^{2}}
$$

where $\eta_{T}$ is the truncation error estimator (the $L^{2}$ norm of the atomistic stress tensor close to the outer boundary), $\eta_{M}$ is the modelling error estimator (the difference of a/c stress tensor and atomistic stress tensor), and $\eta_{C}$ is the coarsening error (jump of a/c stress tensor across interior edges). They are given by

$$
\eta_{T}\left(y_{h}\right):=C_{1}\left\|\sigma^{\mathrm{a}}\left(I_{\mathrm{a}} y_{h}\right)-\sigma^{\mathrm{B}}\right\|_{L^{2}\left(\Omega_{R} \backslash B_{R / 2}\right)},
$$


where $\sigma^{\mathrm{B}}=\frac{1}{\operatorname{det} \mathrm{A}} \sum_{\rho \in \mathcal{R}_{\ell}} \partial_{\rho} V(\mathrm{~B} a) \otimes \rho$.

$$
\begin{aligned}
& \eta_{M}\left(y_{h}\right):=C_{2}\left\{\sum_{T \in \mathcal{T}_{\mathrm{a}}}|T|\left[\sigma^{\mathrm{a}}\left(I_{\mathrm{a}} y_{h}, T\right)-\sum_{T^{\prime} \in \mathcal{T}_{\mathrm{h}}, T^{\prime} \cap T \neq \varnothing} \frac{\left|T^{\prime} \cap T\right|}{|T|} \sigma^{\mathrm{h}}\left(y_{h}, T^{\prime}\right)\right]^{2}\right\}^{\frac{1}{2}}, \\
& \eta_{C}\left(u_{h}\right):=C_{3}\left(\sum_{f \in \mathcal{F}_{h}}\left(h_{f} \llbracket\left[\sigma^{\mathrm{h}} \rrbracket\right)^{2}\right)^{\frac{1}{2}},\right.
\end{aligned}
$$

where $C_{1}, C_{2}$, and $C_{3}$ are independent of $R$ and $u_{h}$, actually $C_{3}=\sqrt{3} C C_{\mathcal{T}_{h}}^{\prime}$ depends only on the shape regularity of $\mathcal{T}_{\mathrm{h}}$.

In this section, we will propose an adaptive mesh refinement algorithm for finite range interactions based on the a posteriori error estimates (5.1). Numerical experiments show that our algorithm achieves a quasi-optimal convergence rate in terms of accuracy vs. the degrees of freedom, which is also consistent with the optimal a priori error estimates.

\subsection{Adaptive mesh refinement algorithm}

We will develop the adaptive mesh refinement algorithm for GRAC method with finite range interactions. In [41], we have designed the adaptive algorithm for GRAC with nearest neighbor interaction. In the nearest neighbor case, there exists a special set of reconstruction parameters [28]. However, with finite range interactions, we have to apply $\ell^{1}$-minimization to solve the reconstruction parameters from (3.3) and (3.6). Furthermore, we need to stabilize the coupling scheme by adding a stabilization term at the interface region, which will effectively add a second order term to the interface stress tensor. We will compare the effects of $\ell^{1}$ minimization and stabilization in numerical experiments.

\subsubsection{Stress tensor correction}

By (4.16)-(4.18), the error estimators $\eta_{T}, \eta_{M}$, and $\eta_{C}$ depend on the stress tensors $\sigma^{\mathrm{h}}$ and $\sigma^{\mathrm{a}}$, which are unique up to divergence free tensor fields by Lemma 4.1. In principle, we need to minimize $\eta\left(y_{h}\right):=\tilde{\eta}\left(\sigma^{\mathrm{a}}\left(I_{\mathrm{a}} y_{h}\right), \sigma^{\mathrm{h}}\left(y_{h}\right)\right)=\eta_{T}\left(y_{h}\right)+\eta_{M}\left(y_{h}\right)+\eta_{C}\left(y_{h}\right)$ with respect to all the admissible stress tensors

$$
\left\langle\delta \mathscr{E}\left(I_{\mathrm{a}} y_{h}\right), v\right\rangle \leq \min _{\mathcal{c}_{a} \in N_{1}\left(\mathcal{T}_{a}\right)^{2}, c_{h} \in N_{1}\left(\mathcal{T}_{h}\right)^{2}} \tilde{\eta}\left(\sigma^{\mathrm{a}}\left(I_{\mathrm{a}} y_{h}\right)+\nabla c_{a} \mathrm{~J}, \sigma^{\mathrm{h}}\left(y_{h}\right)+\nabla c_{h} \mathrm{~J}\right)\|\nabla v\|_{L^{2}} .
$$

To save computational power, we can choose a "good" a/c stress tensor instead of the "optimal" one. A "good" a/c stress tensor should satisfy the following natural conditions:

- Equal to the atomistic stress tensor in the atomistic domain.

- Equal to the continuum stress tensor for uniform deformation. 


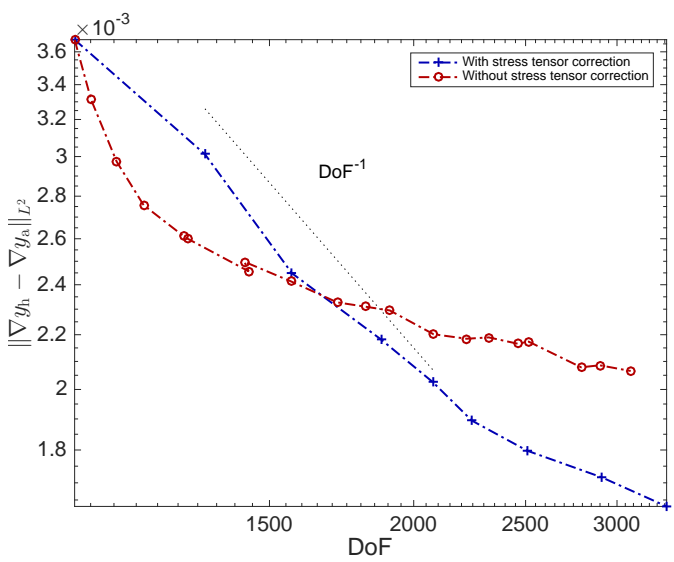

(a) $H^{1}$ error

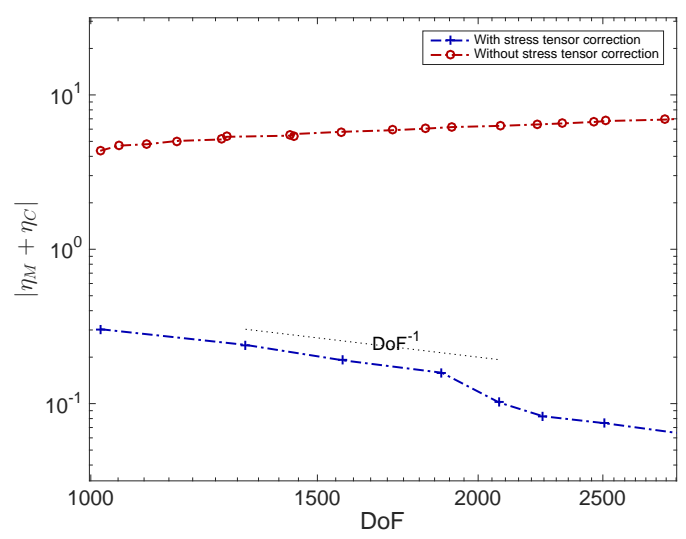

(b) Estimator

Figure 3: Effect of approximate stress tensor correction. Divacancy example. Fig. 3(a): $H^{1}$ error vs. DoF; Fig. 3(b): $\eta_{M}+\eta_{C}$ vs. DoF.

Then, we choose $c_{a} \equiv 0$ and $c_{h}\left(q_{f}\right)=0$ in (5.5), where $q_{f}$ is the midpoint of $f \in \mathcal{F}_{h}, f \cap \Lambda_{i}=$ $\varnothing$. Also, since $\eta_{T}$ and $\eta_{C}$ are higher order contributions to the estimator, we only need to minimize the modeling error $\eta_{M}$ with respect to the degrees of freedom of $\sigma^{\mathrm{h}}$ adjacent to the interface.

We propose the following Algorithm 1 for approximate stress tensor correction:

\section{Algorithm 1 Approximate stress tensor correction}

1. Take $\sigma^{\mathrm{a}}\left(I_{\mathrm{a}} y_{h}\right)$ and $\sigma^{\mathrm{h}}\left(y_{h}\right)$ as the canonical forms in (4.16) and (4.18) respectively.

2. Denote $q_{f}$ as the midpoint of $f \in \mathcal{F}_{h}, f \cap \Lambda_{i} \neq \varnothing$. $c_{h}$ minimizes the following sum

$$
\sum_{T \in \mathcal{T}^{\mathrm{i}}}|T|\left[\sigma^{\mathrm{a}}\left(I_{\mathrm{a}} y_{h}, T\right)-\left(\sigma^{\mathrm{h}}\left(I_{\mathrm{a}} y_{h}, T\right)+\nabla c_{h} \mathrm{~J}\right)\right]^{2}
$$

subject to the constraint that $c_{h}\left(q_{f}\right)=0$, for $f \cap \Lambda_{\mathrm{i}}=\varnothing$.

3. Let $\sigma^{\mathrm{h}}\left(y_{h}\right)=\sigma^{\mathrm{h}}\left(y_{h}\right)+\nabla c^{h} \mathrm{~J}$, compute $\eta_{M}, \eta_{T}$ and $\eta_{C}$ with $\sigma^{\mathrm{a}}\left(I_{\mathrm{a}} y_{h}\right)$ and $\sigma^{\mathrm{h}}\left(y_{h}\right)$.

The effect of the approximate stress tensor correction is demonstrated in Fig. 3. We can see in Fig. 3(a) that the convergence rate is suboptimal if the stress tensor correction is not applied. Fig. 3(b) shows that without correction, the error estimator $\eta$ may even increase with respect to the degree of freedom. 


\subsubsection{Local error estimator}

We can evaluate the global estimator $\eta$ by computing its three components individually. These components correspond to different operations in the adaptive algorithm. The value of truncation error $\eta_{T}$ is determined by the domain size. As the size of the computational domain becomes larger, we have smaller $\eta_{T}$. Hence, we regard $\eta_{T}$ as a criterion to control the domain size. Values of the modelling error $\eta_{M}$ and the coarsening error $\eta_{C}$ indicate local error contributions, and we need to assign $\eta_{M}$ and $\eta_{C}$ to local elements properly.

We define

$$
\eta_{M}\left(T, T^{a}\right):=\left|T^{a} \bigcap T\right|\left[\sigma^{\mathrm{a}}\left(I_{\mathrm{a}} y_{h}, T^{a}\right)-\sum_{T^{\prime} \in \mathcal{T}_{\mathrm{h}}, T^{\prime} \cap T^{a} \neq \varnothing} \frac{\left|T^{\prime} \cap T^{a}\right|}{\left|T^{a}\right|}\left(\sigma^{\mathrm{h}}\left(y_{h}, T^{\prime}\right)\right)\right]^{2} .
$$

Note that $\eta_{M}\left(T, T^{a}\right) \neq 0$ only if $T^{a} \cap \partial T \neq \varnothing$.

For $T^{a} \in \mathcal{T}_{\mathrm{a}}$, then let

$$
\begin{aligned}
& \eta_{M}(T)=\sum_{T^{a} \in \mathcal{T}_{\mathrm{a}}, T^{a} \cap T \neq \varnothing} \eta_{M}\left(T, T^{a}\right), \quad \text { for } T \in \mathcal{T}_{\mathrm{h}}, \\
& \eta_{C}(T)=\sqrt{3} C^{\operatorname{Tr}} C_{\mathcal{T}_{\mathrm{h}}}^{\prime} \sum_{f \in \mathcal{F}_{h} \cap T \in \mathcal{T}_{\mathrm{h}}} \frac{1}{2}\left(h_{f} \llbracket \sigma^{\mathrm{h}} \rrbracket_{f}\right)^{2} .
\end{aligned}
$$

Once all the local estimators are assigned, we are ready to define the indicator $\rho_{T}$ :

$$
\rho_{T}=\left(C^{\operatorname{Tr}}\right)^{2} \frac{\eta_{M}(T)}{\eta_{M}}+\left(\sqrt{3} C^{\operatorname{Tr}} C_{\mathcal{T}_{\mathrm{h}}}^{\prime}\right)^{2} \frac{\eta_{C}(T)}{\eta_{C}} .
$$

Notice that the sum of local estimators $\rho_{T}$ together with truncation error $\eta_{T}$ is equal to the global estimator $\eta$. The constants $C^{\operatorname{Tr}}, C_{\mathcal{T}_{\mathrm{h}}}^{\prime}$ in (5.8) are not known a prior, instead, we use their empirical estimates in the numerical implementation.

Here is the main Algorithm 2, where the Dörfler adaptive strategy [6] is adopted.

\subsection{Numerical experiments}

We present the numerical experiments with the following model problem. We consider the two dimensional triangular lattice $\Lambda^{\text {hom }}:=A \mathbb{Z}^{2}$ with

$$
A=\left[\begin{array}{ll}
1 & \cos (\pi / 3) \\
0 & \sin (\pi / 3)
\end{array}\right]
$$

Let $a_{1}=(1,0)^{T}$, then $a_{j}=\mathrm{A}_{6}^{j-1} a_{1}, j=1, \cdots, 6$, are the nearest neighbor directions in $\Lambda^{\text {hom }}$, where $A_{6}$ is the rotation matrix corresponding to a $\pi / 3$ clockwise planar rotation. Let $\mathcal{N}=\left\{a_{i}\right\}_{i=1}^{6}$ denote the set of nearest neighbor interacting bonds. Given the cut-off radius 


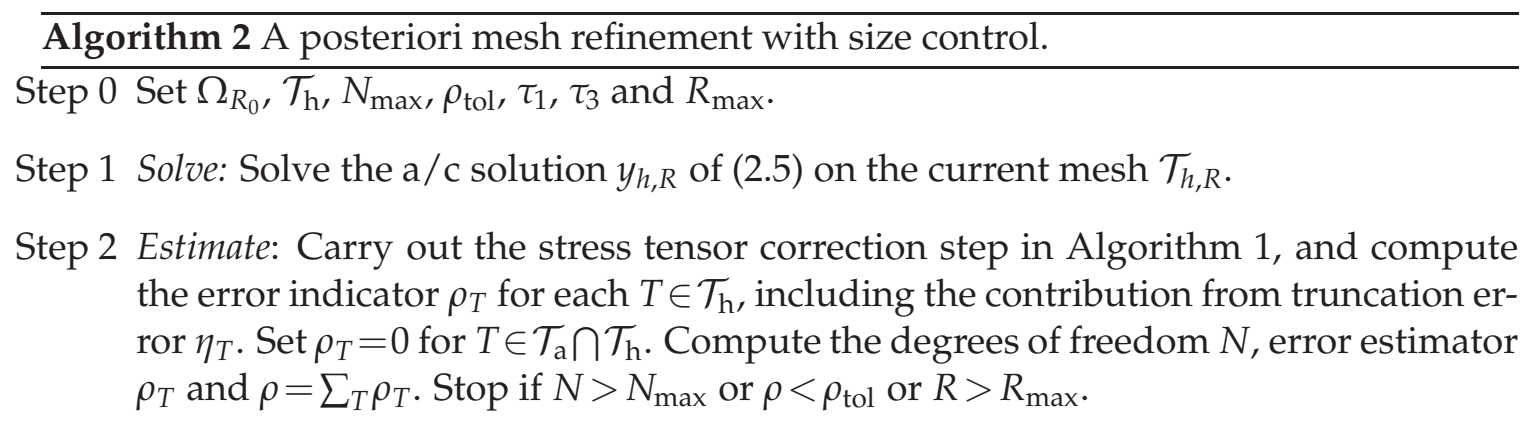

Step 3 Mark:

Step 3.1: Choose a minimal subset $\mathcal{M} \subset \mathcal{T}_{\mathrm{h}}$ such that

$$
\sum_{T \in \mathcal{M}} \rho_{T} \geq \frac{1}{2} \sum_{T \in \mathcal{T}_{\mathrm{h}}} \rho_{T}
$$

Step 3.2: We can find the interface elements which are within $k$ layers of atomistic distance, $\mathcal{M}_{\mathrm{i}}^{k}:=\left\{T \in \mathcal{M} \cap \mathcal{T}_{\mathrm{h}}^{\mathrm{c}}: \operatorname{dist}\left(T, \Lambda^{\mathrm{i}}\right) \leq k\right\}$. Choose $K \geq 1$, find the first $k \leq K$ such that

$$
\sum_{T \in \mathcal{M}_{\mathrm{i}}^{k}} \rho_{T} \geq \tau_{1} \sum_{T \in \mathcal{M}} \rho_{T}
$$

with tolerance $0<\tau_{1}<1$. If such a $k$ can be found, let $\mathcal{M}=\mathcal{M} \backslash \mathcal{M}_{\mathrm{i}}^{k}$. Then in step 3, expand interface $\Lambda_{\mathrm{i}}$ outward by $k$ layers.

Step 4 Refine: If (5.9) is true, expand interface $\Lambda_{\mathrm{i}}$ outward by one layer. If $\eta_{T} \geq \tau_{3} \rho$, enlarge the computational domain. Bisect all elements $T \in \mathcal{M}$. Stop if $\frac{\eta_{T}}{\eta_{M}+\eta_{C}} \geq \tau_{2}$, otherwise, go to Step 1.

$r_{\text {cut }}$, then each interaction direction $\rho \in \mathcal{R}$ can be uniquely represented as $\rho=\alpha a_{i}+\beta a_{i+1}$, where $\alpha \geq 0, \beta \geq 0, \alpha+\beta \leq r_{\text {cut }}$ and $a_{i} \in \mathcal{N}$.

Recall the EAM potential defined in (2.1). Let

$$
\begin{aligned}
& \phi(r)=\exp (-2 a(r-1))-2 \exp (-a(r-1)), \quad \psi(r)=\exp (-b r), \\
& F(\tilde{\rho})=C\left[\left(\tilde{\rho}-\tilde{\rho}_{0}\right)^{2}+\left(\tilde{\rho}-\tilde{\rho}_{0}\right)^{4}\right],
\end{aligned}
$$

with parameters $a=4.4, b=3, c=5$ and $\tilde{\rho}_{0}=6 \exp (-b)$, which is the same as the numerical experiments in the a priori analysis of GRAC method [29].

To generate a defect, we remove $k$ atoms from $\Lambda^{\text {hom }}$,

$$
\begin{aligned}
& \left.\Lambda_{k}^{\text {def }}:=\left\{-(k / 2) e_{1}, \cdots,(k / 2-1) e_{1}\right)\right\}, \quad \text { if } k \text { is even, } \\
& \left.\Lambda_{k}^{\text {def }}:=\left\{-(k-1) / 2 e_{1}, \cdots,(k-1) / 2 e_{1}\right)\right\}, \quad \text { if } k \text { is odd, }
\end{aligned}
$$


and $\Lambda=\Lambda^{\text {hom }} \backslash \Lambda_{k}^{\text {def }}$

For $\ell \in \Lambda$, consider the next nearest neighbor interaction, $\mathcal{N}_{\ell}:=\left\{\ell^{\prime} \in \Lambda|0<| \ell^{\prime}-\ell \mid \leq 2\right\}$, and interaction range $\mathcal{R}_{\ell}:=\left\{\ell^{\prime}-\ell \mid \ell^{\prime} \in \mathcal{N}_{\ell}\right\} \subseteq\left\{a_{j}, j=1, \cdots, 18\right\}$. The defect core $D^{\text {def }}$ can be defined by $D^{\text {def }}=\left\{x: \operatorname{dist}\left(x, \Lambda_{k}^{\text {def }}\right) \leq 2\right\}, \Lambda \cap D^{\text {def }}$ is the first layer of atoms around $\Lambda_{k}^{\text {def }}$.

\subsubsection{Di-vacancy}

In this section, we numerically justify the performance of the proposed adaptive mesh refinement algorithm. We take the same di-vacancy example in [29], namely, setting $k=2$ for $\Lambda_{k}^{\text {def. }}$. We apply isotropic stretch $S$ and shear $\gamma_{I I}$ by setting

$$
B=\left(\begin{array}{cc}
1+S & \gamma_{I I} \\
0 & 1+S
\end{array}\right) \cdot F_{0}
$$

where $F_{0} \propto I$ minimizing the Cauchy-Born energy density $\mathrm{W}, \mathrm{S}=\gamma_{I I}=0.03$.

\subsubsection{Micro-crack}

In the microcrack experiment, we remove a longer segment of atoms, $\Lambda_{11}^{\text {def }}=$ $\left\{-5 e_{1}, \cdots, 5 e_{1}\right\}$ from the computational domain. The body is then loaded in mixed mode I \& II, by setting

$$
\mathrm{B}:=\left(\begin{array}{cc}
1 & \gamma_{\mathrm{II}} \\
0 & 1+\gamma_{\mathrm{I}}
\end{array}\right) \cdot \mathrm{F}_{0}
$$

where $F_{0} \propto I$ minimizes $W$, and $\gamma_{I}=\gamma_{I I}=0.03$ ( $3 \%$ shear and $3 \%$ tensile stretch).

We take $\tau_{3}=0.7$ in the numerical implementation of Algorithm 2. We compare results with and without stabilisation and also with different optimisation approaches to obtain the reconstruction parameters.

From the numerical results in Figs. 4-7, we can see that the least square method for the reconstruction parameters does not converge at all. For the $\ell^{1}$-minimization approach without stabilization, there exist large errors in the pre-asymptotic regime, though the solutions tend to converge with increasing degree of freedom. For $\ell^{1}$-minimization combined with stabilisation, we are able to obtain the quasi-optimal convergence rate that consistent with the a priori result.

Fig. 8 shows the computational time for the main components of the algorithm with respect to degrees of freedom. The current implementation, we strictly stick to the rigorous a posteriori estimate. The dominated cost is to find those triangles $T^{a} \in \mathcal{T}_{\text {a }}$ such that $T^{a} \cap \partial T \neq \varnothing$ for $T \in \mathcal{T}_{\mathrm{h}}$ in order to compute $\eta_{M}\left(T, T^{a}\right)$ in (5.7). We are currently working on the reduction of this cost. Furthermore, the contribution of $\eta_{M}$ is actually second order away from the interface, in practical computation this part could be neglected. Except the computational cost for the estimator $\eta$, which will be optimized in the future implementation, the a priori algorithm in [29] and the a posteriori algorithm here have similar running time. 


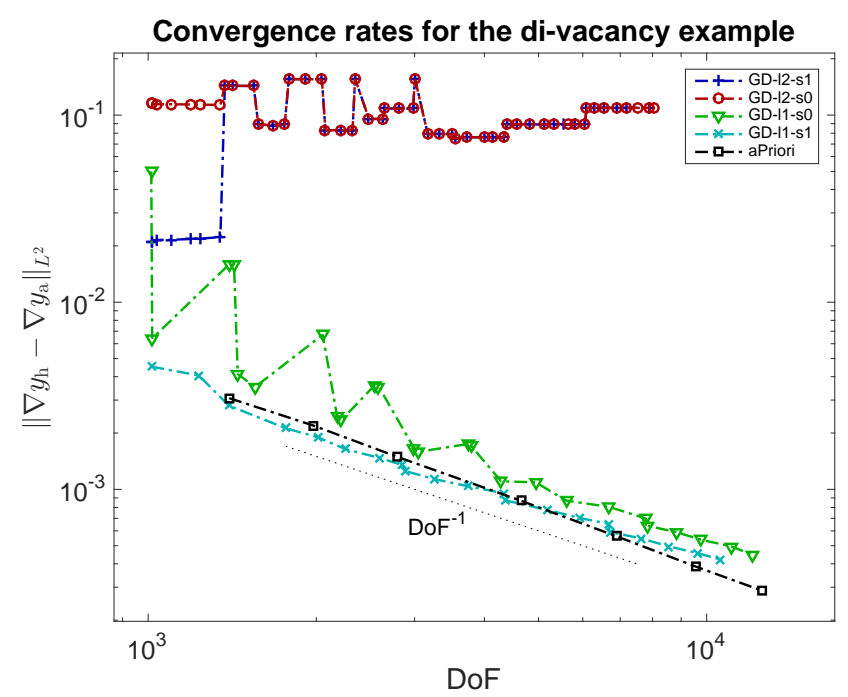

Figure 4: Numerical results by Algorithm 2: $H^{1}$ error vs. $N$, using a posteriori estimator in $H^{1}$ norm. In the legend, I1 means $\ell^{1}$-minimization approach while 12 represents a least-squares approach; s1 indicates with stabilization and s0 without.

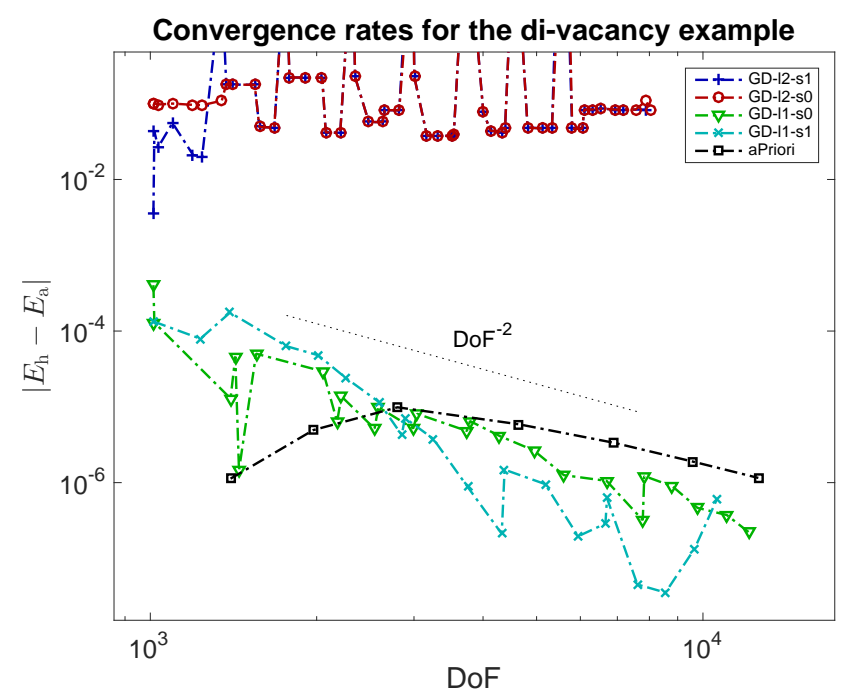

Figure 5: Numerical results by Algorithm 2: Energy error vs. $N$, using a posteriori estimator in $H^{1}$ norm. In the legend, I1 means $\ell^{1}$-minimization approach while 12 represents a least-squares approach; s1 indicates with stabilization and $\mathrm{s} 0$ without.

Fig. 9 shows the ratio between the a posteriori error estimator and true error for the di-vacancy example, which is about $50-100$. For practical implementations, the refinement indicator may also be used to control the adaptivity, which will be left for future investigation. 


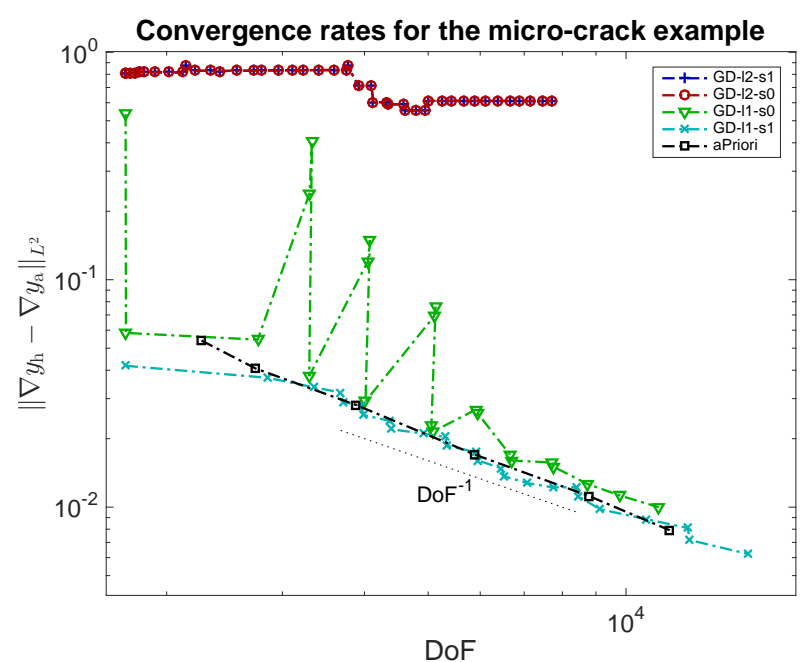

Figure 6: Numerical results by Algorithm 2: $H^{1}$ error vs. $N$, using a posteriori estimator in $H^{1}$ norm. In the legend, 11 means $\ell^{1}$-minimization approach while 12 represents a least-squares approach; s1 indicates with stabilization and s0 without.

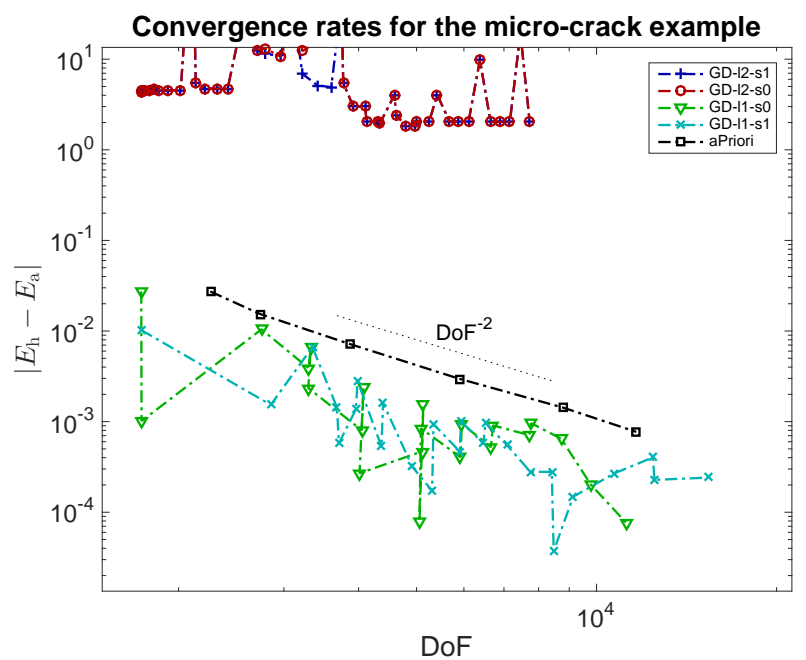

Figure 7: Numerical results by Algorithm 2: Energy error vs. $N$, using a posteriori estimator in $H^{1}$ norm. In the legend, I1 means $\ell^{1}$-minimization approach while 12 represents a least-squares approach; s1 indicates with stabilization and s0 without.

Remark 5.1. The time of the energy minimization depends on the applied iteration method, iteration loops and tolerance. The two peaks in Fig. 8 takes about ten times the steps to reach the given tolerance $10^{-7}$ by line search. In our numerical experiments, we could applied trust region method after line search to obtain $10^{-10}$ accuracy, but the time it costs will approach that of $\sigma_{a}$. 


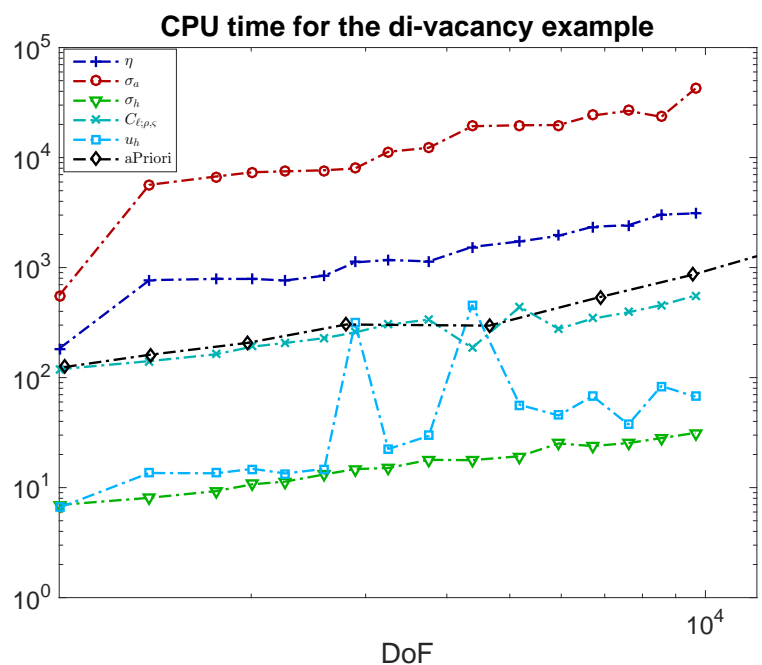

Figure 8: CPU time of the divacancy example, $\eta$ : the time to compute the estimator $\eta ; \sigma_{a}$ : the time to compute the atomistic stress $\sigma^{\text {a }}$ on those triangles in $T^{\text {a }}$ which intersect with edges in $T^{h} ; \sigma_{h}$ : the time to compute $\sigma_{h}$; $C_{\ell ; \rho, \zeta}$ : the time to compute reconstruction parameters with $\ell^{1}$ minimization; $u_{h}$ : the time for the minimization of the coupling energy; aPriori: the time for the a priori computation of the a/c solution.

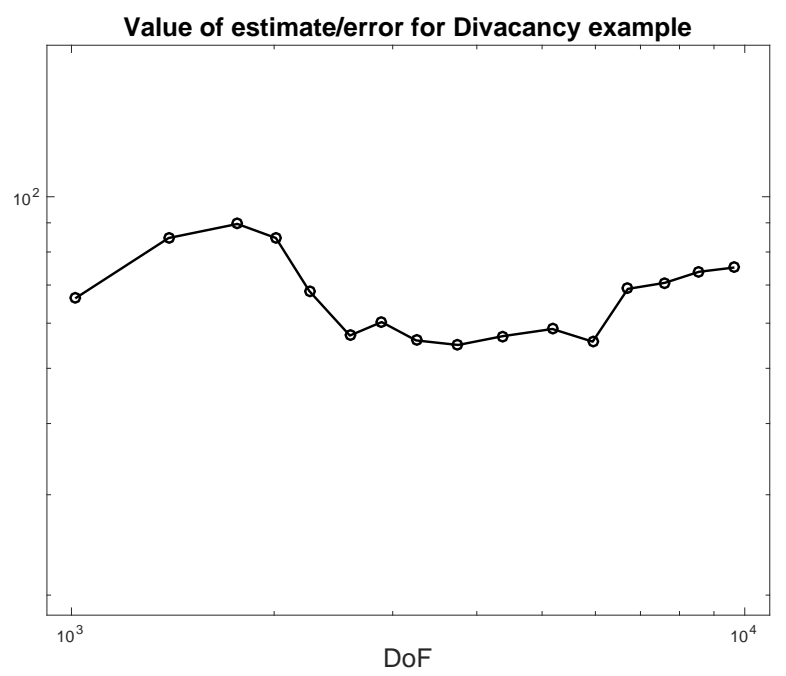

Figure 9: Ratio (in $\log _{10}$ scale) of error estimate and error vs. DoF for divacancy example.

\section{Conclusion}

In this paper, we construct the adaptive algorithm for a class of consistent (ghost force free) atomistic/continuum coupling schemes with finite range interactions based on the rigorous a posteriori error estimates. Different from the localization formula for the stress tensor in the a priori analysis $[21,22,26]$, we develop a computable formulation for the 
stress tensor. Combined with $\ell_{1}$-minimization approach for reconstruction parameters and stabilization, we have shown that the numerical results for the corresponding adaptive algorithms are comparable to quasi-optimal a priori analysis.

The extension to three dimensional problems is not trivial, it require an efficient combination of the a/c coupling method and error estimation. Currently, the construction of the consistent 3D GRAC coupling is in progress. We are also working on the a posteriori error estimates of the blending method and the BGFC method [30], which would be more flexible for the extension to 3D. The stress formulation in this paper can be extended to $3 \mathrm{D}$, though it requires to compute the intersection of interaction bonds with elements, it could be done in 3D but will be complicated. As in [34,35], we will carefully consider the application of bond-density-lemma to simplify the computation. Furthermore, the approximate stress tensor correction can be done locally, similar to the local flux reconstruction in [31].

More practical problems, for example, the study of dislocation nucleation and dislocation interaction by a/c coupling methods has attracted considerable attention from the early stage of a/c coupling methods $[32,38]$. The difficulty is to deal with boundary condition and complicated geometry changes of the interface.

For general atomistic/continuum coupling schemes, such as BQCE, BQCF and BGFC, the a priori analysis in $[13,15,30]$ provide a general analytical framework and the stress tensor based formulation plays a key role in the analysis. Therefore, the a posteriori analysis for those coupling schemes can inherit this analytical framework and the stress tensor formulation. Techniques developed in this paper will be essential for the efficient implementation of the corresponding adaptive algorithms.

\section{Acknowledgments}

ML and PL were partially supported by National Natural Science Foundation of China grant 11861131004, 11771040, 91430106. LZ was partially supported by Natural Science Foundation of China grant 11871339, 11861131004, 11571314, 11471214 and the One Thousand Plan of China for young scientists.

\section{A Appendix}

\section{A.1 Derivation of (4.8) for two dimensional triangular lattice}

In this section, for two dimensional triangular lattice introduced in Section 5.2, we derive (4.8). We first classify interaction bonds into two types according to their intersection to corresponding elements.

Type I interaction bonds is parallel to one of the nearest neighbor directions. The set of all Type I bonds is defined by $\mathrm{B}_{\mathrm{I}}=\left\{\rho \mid \exists \mathrm{a}_{i} \in \mathcal{R}_{\text {nn }}\right.$, such that $\left.\rho=|\rho| a_{i}\right\}$, as illustrated in Fig. 10. 


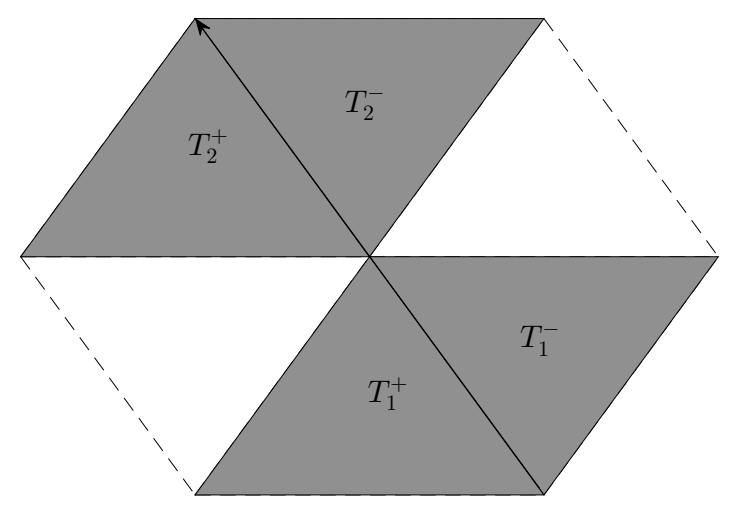

Figure 10: Illustration of corresponding elements (marked gray) to bond $\rho \in \mathrm{B}_{\mathrm{I}}$ with $\rho=2 a_{3}$.

For each $\rho \in \mathrm{B}_{\mathrm{I}}$ with start point $\ell \in \Lambda$ and $v \in \mathscr{U}^{1,2}$, we have

$$
\begin{aligned}
D_{\rho} v & =\sum_{k=1}^{|\rho|} D_{a_{i}} v\left(\ell+(k-1) a_{i}\right) \\
& =\left(\sum_{k=1}^{|\rho|}\left(\frac{1}{2} \nabla_{T_{k}^{+}} v+\frac{1}{2} \nabla_{T_{k}^{-}} v\right)\right) \cdot \frac{\rho}{|\rho|} \\
& =\sum_{k=1}^{|\rho|} \frac{1}{2|\rho|}\left(\nabla_{T_{k}^{+} v} \cdot \rho+\nabla_{T_{k}^{-}} v \cdot \rho\right),
\end{aligned}
$$

where $T_{k}^{+}$and $T_{k}^{-}$are the triangles with $\left(\ell+(k-1) a_{i}, \ell+k a_{i}\right)$ as the common edge, for $k=1, \cdots,|\rho|$, superscript " + " means the element being located on the left of the bond. In this case, the contribution in $(4.8)$ is

$$
\omega_{\ell}^{\rho}\left(T_{k}^{( \pm)}\right)=\frac{1}{2|\rho|}
$$

Type II interaction bonds is not parallel to any of the nearest neighbor directions, therefore they must cross the intersecting elements. The set of all Type II bonds is defined by $\mathrm{B}_{\mathrm{II}}=\left\{\rho \mid \rho\right.$ is not parallel to any $\left.a_{i} \in \mathcal{R}_{\mathrm{nn}}\right\}$, as illustrated in Fig. 11 .

For any Type II interaction bond $\rho \in \mathrm{B}_{\mathrm{II}}$, there exists nearest neighbor directions $a_{i}$, $a_{i+1}$, such that $\rho=\alpha a_{i}+\beta a_{i+1}, \alpha, \beta>0$. Let $(\ell, \ell+\rho)$ consecutively intersect with elements $T_{k}, k=1, \cdots, n_{p}$, for $v \in \mathscr{U}^{1,2}$, we have

$$
\begin{aligned}
D_{\rho} v & =\sum_{k=1}^{n_{\rho}} D_{\rho_{k}} v\left(\ell+\sum_{t=1}^{k} \rho_{k-1}\right)=\left(\sum_{k=1}^{n_{\rho}} \nabla_{T_{k}} v\right) \cdot \rho_{k} \\
& =\left(\sum_{k=1}^{n_{\rho}} \nabla_{T_{k}} v\right) \cdot \frac{\left|\rho_{k}\right|}{|\rho|} \rho=\sum_{k=1}^{\left.n_{\rho}\right)} \frac{\left|\rho_{k}\right|}{|\rho|} \nabla_{T_{k}} v \cdot \rho,
\end{aligned}
$$




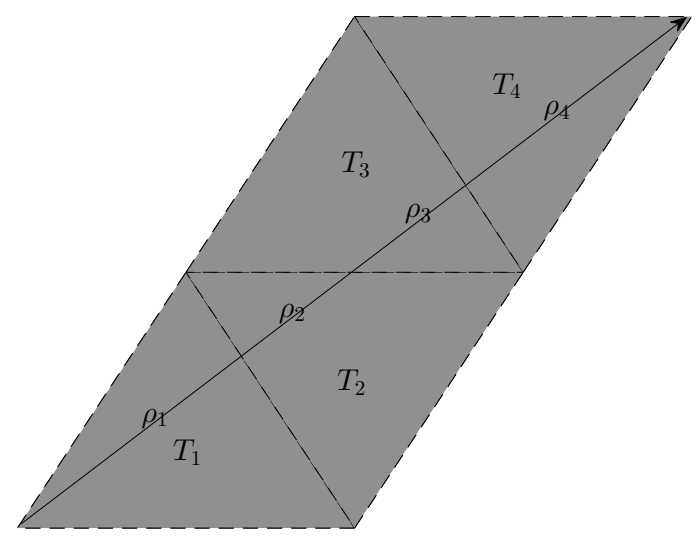

Figure 11: Illustration of the intersecting elements (marked gray) with respect to bond $\rho \in \mathrm{B}_{\mathrm{II}}, \rho=a_{1}+2 a_{2}$.

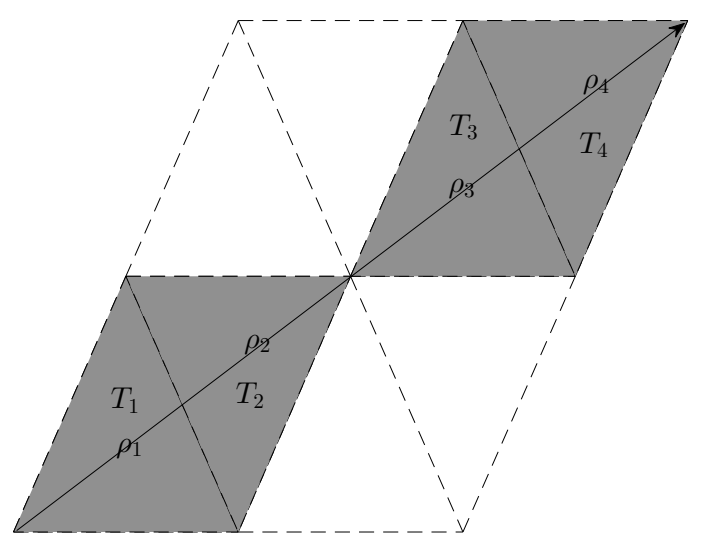

Figure 12: Illustration of corresponding elements (marked gray) to bond $\rho \in \mathrm{B}_{\mathrm{I}}$ with $\rho=2 a_{1}+2 a_{2}$.

where the set of vectors $\left\{\rho_{k}\right\}_{k=1}^{n_{\rho}}$ is the unit partition of $\rho$ with $\rho=\sum_{k=1}^{n_{\rho}} \rho_{k}, \rho_{0}=\mathbf{0}$ and $\frac{\rho_{k}}{\left|\rho_{k}\right|}=\frac{\rho}{|\rho|}$ for $k=1, \cdots, n_{p} \cdot \rho_{k}=T_{k} \cap(\ell, \ell+\rho)$. For the two dimensional triangular lattice as in Section 5.2, we have $n_{\rho}=2(\alpha+\beta-1)$ if $\alpha \neq \beta$, and $n_{\rho}=2 \alpha$ if $\alpha=\beta$. In this case,

$$
\omega_{\ell}^{\rho}\left(T_{k}\right)=\frac{\left|\rho_{k}\right|}{|\rho|} .
$$

For the Type II bonds with $\alpha=\beta$, in the hexagon site geometry, The contribution factor $\omega_{\rho}^{T}$ is the same for every element related to bond $\rho$ with the value $\omega_{\rho}^{T}=\frac{1}{2 \alpha}$ which can be see from Fig. 12.

\section{References}

[1] M. Arndt and M. Luskin. Goal-oriented adaptive mesh refinement for the quasicontinuum approximation of a Frenkel-Kontorova model. Comput. Methods Appl. Mech. Engrg., 197(49):4298-4306, 2007. 
[2] M. Arndt and M. Luskin. Error estimation and atomistic-continuum adaptivity for the quasicontinuum approximation of a Frenkel-Kontorova model. Multiscale Model. Simul., 7(1):147-170, 2008.

[3] H. Chen and C. Ortner. Qm/mm methods for crystalline defects. part 1: Locality of the tight binding model. Multiscale Model. Simul., 14(1):232-264, 2016.

[4] H. Chen and C. Ortner. Qm/mm method for crystalline defects. part 2: Consistent energy and force-mixing. Multiscale Model. Simul., 15(1):184-214, 2017.

[5] M. S. Daw and M. I. Baskes. Embedded-atom method: Derivation and application to impurities, surfaces, and other defects in metals. Phys. Rev. B, 29(12):6443-6453, 1984.

[6] W. Dörfler. A convergent adaptive algorithm for poisson's equation. SIAM J. Numer. Anal., 33(3):1106-1124, 1996.

[7] W. E, J. Lu, and J. Z. Yang. Uniform accuracy of the quasicontinuum method. Phys. Rev. B, $74(21): 214115,2006$.

[8] W. E and P. Ming. Cauchy-Born rule and the stability of crystalline solids: static problems. Arch. Ration. Mech. Anal., 183(2):241-297, 2007.

[9] V. Ehrlacher, C. Ortner, and A. V. Shapeev. Analysis of boundary conditions for crystal defect atomistic simulations. Arch. Rat. Mech. Anal., 222(3):1217-1268, 2016.

[10] L. Fang, A. Majumdar, and L. Zhang. Multiscale numerical method for nematic liquid crystal, 2018. Work in progress.

[11] M. W. Finnis and J. E. Sinclair. A simple empirical n-body potential for transition-metals. Philos. Mag. A, 50(1):45-55, 1984.

[12] H. Fischmeister, H. Exner, M. H. Poech, S. Kohlhoff, P. Gumbsch, S. Schmauder, L. S. Sigi, and R. Spiegler. Modelling fracture processes in metals and composite materials. $\underline{Z}$. Metallkde., 80(12):839-846, 1989.

[13] X. H. Li, C. Ortner, A. V. Shapeev, and B. Van Koten. Analysis of blended atomistic/continuum hybrid methods. Numer. Math., 134(2):275-326, 2015.

[14] P. Lin. Convergence analysis of a quasi-continuum approximation for a two-dimensional material without defects. SIAM J. Numer. Anal., 45(1):313-332, 2007.

[15] J. Lu and P. Ming. Convergence of a force-based hybrid method in three dimension. Comm. Pure Appl. Math., 66(1):83-108, 2013.

[16] M. Luskin and C. Ortner. Atomistic-to-continuum coupling. Acta Numer., 22(22):397-508, 2013.

[17] M. Luskin, C. Ortner, and B. V. Koten. Formulation and optimization of the energy-based blended quasicontinuum method. Comput. Methods Appl. Mech. Engrg., 253(1):160-168, 2013.

[18] R. E. Miller and E. B. Tadmor. A unified framework and performance benchmark of fourteen multiscale atomistic/continuum coupling methods. Modelling Simul. Mater. Sci. Eng., 17(5):053001, 2009.

[19] M. Ortiz, R. Phillips, and E. B. Tadmor. Quasicontinuum analysis of defects in solids. Philos. Mag. A, 73(6):1529-1563, 1996.

[20] C. Ortner. A priori and a posteriori analysis of the quasi-nonlocal quasicontinuum method in 1D. Math. Comp., 80(275):1265-1285, 2011.

[21] C. Ortner. The role of the patch test in $2 \mathrm{~d}$ atomistic-to-continuum coupling methods. ESAIM Math. Model. Numer. Anal., 46(6):1275-1319, 2011.

[22] C. Ortner and A. V. Shapeev. Interpolation of lattice functions and applications to atomistic/continuum multiscale methods. ArXiv e-prints, arXiv:1204.3705, 2012.

[23] C. Ortner and A. V. Shapeev. Analysis of an energy-based atomistic/continuum coupling 
approximation of a vacancy in the 2d triangular lattice. Math. Comp., 82:2191-2236, 2013.

[24] C. Ortner, A. V. Shapeev, and L. Zhang. (in-)stability and stabilisation of qnl-type atomisticto-continuum coupling methods. SIAM Multiscale Model. Simul., 12(3):1258-1293, 2014.

[25] C. Ortner and E. Süli. Analysis of a quasicontinuum method in one dimension. Math. Model. Numer. Anal., 42(1):57-91, 2008.

[26] C. Ortner and F. Theil. Justification of the cauchy-born approximation of elastodynamics. Arch. Ration. Mech. Anal., 207(3):1025-1073, 2013.

[27] C. Ortner and H. Wang. A posteriori error control for a quasi-continuum approximation of a periodic chain. IMA J. Numer. Anal., 34(3):977-1001, 2014.

[28] C. Ortner and L. Zhang. Construction and sharp consistency estimates for atomistic/continuum coupling methods with general interfaces: a $2 \mathrm{~d}$ model problem. SIAM J. Numer. Anal., 50(6):2940-2965, 2012.

[29] C. Ortner and L. Zhang. Energy-based atomisitic-to-continuum coupling without ghost forces. Comput. Methods Appl. Mech. Engrg., 279(1):29-45, 2014.

[30] C. Ortner and L. Zhang. Atomistic/continuum blending with ghost force correction. SIAM J. Sci. Comput., 38(1):A346-A375, 2016.

[31] J. Papež, Z. Strakoš, and M. Vohralík. Estimating and localizing the algebraic and total numerical errors using flux reconstructions. Numer. Math., 3(138):681-214, 2018.

[32] R. Phillips, D. Rodney, V. Shenoy, E. B. Tadmor, and M. Ortiz. Hierarchical models of plasticity: dislocation nucleation and interaction. Modelling Simul. Mater. Sci. Eng., 7(5):769-780, 1999.

[33] S. Prudhomme, P. T. Bauman, and J. T. Oden. Error control for molecular statics problems. International Journal for Multiscale Computational Engineering, 4(5-6):647-662, 2006.

[34] A. V. Shapeev. Consistent energy-based atomistic/continuum coupling for two-body potentials in one and two dimensions. Multiscale Model. Simul., 9(3):905-932, 2011.

[35] A. V. Shapeev. Consistent energy-based atomistic/continuum coupling for two-body potentials in three dimensions. SIAM J. Sci. Comput., 34(3):B335-B360, 2012.

[36] V. B. Shenoy, R. E. Miller, E. B. Tadmor, D. Rodney, R. Phillips, and M. Ortiz. An adaptive finite element approach to atomic-scale mechanics-the quasicontinuum method. J. Mech. Phys. Solids, 47(3):611-642, 1999.

[37] T. Shimokawa, J. J. Mortensen, J. Schiotz, and K. W. Jacobsen. Matching conditions in the quasicontinuum method: Removal of the error introduced at the interface between the coarse-grained and fully atomistic region. Phys. Rev. B, 69(21):214104, 2004.

[38] E. B. Tadmor, R. Miller, R. Phillips, and M. Ortiz. Nanoindentation and incipient plasticity. J. Mater. Res., 14(6):2233-2250, 1999.

[39] E. B. Tadmor and R. E. Miller. Modeling Materials: Continuum, Atomistic and Multiscale Techniques. Cambridge University Press, 2012.

[40] I. Tembhekar, J. S. Amelang, L. Munk, and D. M. Kochmann. Automatic adaptivity in the fully-nonlocal quasicontinuum method for coarse-grained atomistic simulations. Int. J. Numer. Meth. Engng., 110(9):878-900, 2017.

[41] H. Wang, M. Liao, P. Lin, and L. Zhang. A posteriori error estimation and adaptive algorithm for the atomistic/continuum coupling in two dimensions. SIAM J. Sci. Comput., 40(4):A2087-A2119, 2018. 